
\title{
R Research Square \\ The total factor productivity index of freshwater aquaculture in China: based on regional heterogeneity
}

\section{Shen Zhong}

Harbin University of Commerce

Aizhi Li

Harbin University of Commerce

Jing Wu (D 102243@hrbcu.edu.cn )

Harbin University of Commerce

\section{Research Article}

Keywords: Freshwater aquaculture, Meta-frontier Malmquist index, Total factor productivity, Sustainable development, Coastal region, Inland region

Posted Date: April 21st, 2021

DOl: https://doi.org/10.21203/rs.3.rs-404345/v1

License: (c) (i) This work is licensed under a Creative Commons Attribution 4.0 International License. Read Full License

Version of Record: A version of this preprint was published at Environmental Science and Pollution Research on October 11th, 2021. See the published version at https://doi.org/10.1007/s11356-02116504-8. 
1 Title: The total factor productivity index of freshwater aquaculture in China: based on

2 regional heterogeneity

3 Author names: Shen $\mathrm{Zhong}^{\mathrm{a}}$, Aizhi $\mathrm{Li}^{\mathrm{a}}$, Jing $\mathrm{Wu}^{\mathrm{a}, *}$

$4 \quad{ }^{a}$ Harbin University of Commerce, Harbin, Heilongjiang, China

5 E-mail addresses: 102714@hrbcu.edu.cn (Shen Zhong), liaizhi@hrbcu.edu.cn (Aizhi

6 Li), 102243@hrbcu.edu.cn (Jing Wu).

7 *Corresponding author: Jing Wu. Tel.: +86-1394-566-0550.

8 Present address: Harbin University of Commerce, No.1 Xuehai Street, Songbei

9 District, Harbin 430072, Heilongjiang, PR China.

Ethics approval and consent to participate: Not applicable.

Consent for publication: Not applicable.

Availability of data and materials: The datasets used and/or analysed during the current study are available from the corresponding author on reasonable request.

Competing interests: The authors declare that they have no competing interests.

Funding: This work was supported by the National Social Science Foundation Project of China (No. 18JL094).

Authors' contributions: Shen Zhong: Conceptualization, Methodology, Software. Aizhi Li: Investigation, Data curation, Formal analysis, Writing-Original draft. Jing Wu: Validation, Writing-Reviewing and Editing.

Acknowledgements: We thank the funding supported by National Social Science Foundation of China (No. 18JL094). And the authors are also grateful to anonymous referees for providing helpful comments and suggestions to improve this study. 


\section{Abstract}

sustainable development of freshwater aquaculture, which is crucial to ensure fish food supply. In particular, finding ways to improve the freshwater aquaculture efficiency, by measuring and analyzing the total factor productivity (TFP) of freshwater aquaculture, is of great significance to the sustainable development of freshwater aquaculture industry. Therefore, this paper constructs a meta-frontier Malmquist index (MMI) model by considering the regional heterogeneity to evaluate the TFP of freshwater aquaculture of China from 2004 to 2019. The results show that: (1) From 2004 to 2019, the TFP of freshwater aquaculture shows dynamic fluctuations and spatial differences. Compared with coastal regions, there are obvious advantages in TFP of freshwater aquaculture in inland regions. (2) The change of TFP in freshwater aquaculture is driven by the combined effect of technology change (TC) and technical efficiency change (EC).

(3) The technology gap in freshwater aquaculture of coastal regions is tiny, while that of inland regions is large. This paper finally gives some policy suggestions to improve the TFP to ensure the sustainable development of freshwater aquaculture.

Keywords: Freshwater aquaculture; Meta-frontier Malmquist index; Total factor productivity; Sustainable development; Coastal region; Inland region 


\section{Introduction}

As an important food for human daily consumption, fish provides people with indispensable amino acids and protein (Merino et al., 2012). China is the main producer of farmed edible fish and has produced more of such edible fish than other countries combined every year since 1991(FAO, 2018). Edible fish plays an increasingly important role in China's food supply (Troell et al., 2014). To ensure the demand of residents for fish food, it is significant to continuously improve the efficiency of fishery culture (Muir, 2005). As the main production mode of fishery aquaculture, freshwater aquaculture efficiency measurement is of great significance to improve the efficiency of aquaculture and ensuring the aquatic biomass. Hence, this paper will measure and analyze the total factor productivity of freshwater aquaculture in China, and get the sustainable development strategy of freshwater aquaculture through the analysis of the efficiency.

There are significant differences in the development of freshwater aquaculture in different regions. For example, there are obvious differences between coastal regions and inland regions in training intensity, technology level, technology promotion and management level of freshwater aquaculture (Ji and Wang, 2015). All of these may affect freshwater aquaculture efficiency. The level of human capital, fishery infrastructure, resource endowment and aquaculture scale will also affect the efficiency of freshwater aquaculture. In addition, coastal regions attach great importance to marine fisheries. In the later stage, with the gradual implementation of the policy of "expanding freshwater and offshore aquaculture", coastal regions gradually paid attention to 
freshwater aquaculture and made great achievements. It is worth noting that there is no research to manifest whether the coastal areas attach importance to mariculture will influence freshwater aquaculture efficiency. Therefore, considering the regional heterogeneity, this paper uses directional distance function (DDF) and MMI model to analyze the total factor productivity of freshwater aquaculture in China from 2014 to 2019. Obviously, it will provide a powerful reference for the sustainable and coordinated development of freshwater aquaculture biomass to China.

The remainder of this study is as follows: The next section introduces the related literature. Section 3 describes the designed methodology applied in the study, the inputoutput variables and data sources. The presentation of empirical analysis is elaborated in Section 4. The main conclusions and policy suggestions are provided in Section 5. And there are many abbreviations are showed in Table 1.

\section{Literature reviewed}

TFP refers to the part of output that is not explained by input. It is a representative index to measure production efficiency (Lin and Ge, 2019). Squire (1992) first applied TFP to fishery, which made fishery research method and system more scientific and provided strong support for many scholars in fishery research. By combing the existing literature, it can be find that the common methods of measuring TFP are stochastic frontier analysis (SFA) and data envelopment analysis (DEA).

First, SFA was introduced by Aigner et al. (1977) and Meeusen and Van den Broeck (1977), which uses the metrology method to measure the growth structure. It is necessary to preset the production function, separate statistical errors and technical 
inefficiencies during the measurement process (Battese and Coelli, 1995; Kumbhakar and Lovell, 2000). Iinuma et al. (1999) measured the technical efficiency of carp pond culture in the Malaysian archipelago by SFA method. Sharma and Leung (2000) applied a stochastic frontier production model to test and explain the differences in carp pond culture techniques among the major carp producing countries in South Asia (India, Bangladesh, Pakistan, and Nepal). Based on the cross-sectional data of 150 fish farms, Onumah et al. (2010) used stochastic frontier function to calculate the technical efficiency and its influencing factors of Ghana fish farms. It is found that the average output adjustment of input is positive. Moreover, the return to scale of fish farms is on the rise. Kim et al. (2011) estimated the production efficiency of sandfish coastal gillnet fishery on the east coast of South Korea with translog stochastic production frontier. Jamnia et al. (2013) employed Cobb-Douglas stochastic production frontier model to measure the technical efficiency of fishery in Chabahar region in southern Iran, and discussed the output elasticity and return to scale. At the same time, their research also found that the translog function cannot be used as an appropriate representation of data. Tingley et al. (2005) applied SFA and DEA method to measure the technical efficiency of the channel fishery, and analyzed the main factors affecting the technical efficiency. Finally, the conclusion is drawn that the two methods are basically the same in the analysis of technical efficiency. When the stochastic production frontier function is difficult to determine accurately, it can be replaced by DEA method. In other words, SFA has some defects. It cannot reasonably control the input-output variables and endogenous problems, and has high requirements for the setting of production function. 
Once the production function is set unreasonably, the result will be biased (Simar and Wilson, 1998).

Second, DEA model constructs nonparametric piecewise surface of data by linear programming, and then calculates the relative effectiveness of multiple input-output decision-making units (DMUs) (Charnes et al., 1978; Coelli, 1998). Compared with SFA model, DEA does not need to calculate any function forms related to input and output (Song and Zheng, 2016), which can avoid strong hypothesis bias such as production function setting and random interference normal distribution (Färe et al., 1989). For this reasons, DEA model has more advantages in evaluating efficiency. Sharma et al. (1999) applied DEA method to measure the economic efficiency and technical efficiency of Chinese fish farms and showed that the low production efficiency of fish farms in China is mainly caused by the low technical efficiency. Rasmus (2011) analyzed the technical efficiency of aquaculture in Denmark with CCRDEA model. Pascoe and Herrero (2004) pointed out that the fishery production function needs to consider the stock, so it is difficult to set. Through research, they proposed that the use of DEA Malmquist productivity index model can avoid the consideration of stock. After verifying the effectiveness, Pascoe used this method to study the fishery production efficiency in the South Atlantic of Spain. Hassanpur et al. (2011) estimated the TFP of rainbow trout aquaculture in Iran by Malmquist index. It is found that the TFP growth of rainbow trout aquaculture in aquaculture sector is mainly caused by the progress of technical efficiency, while the effect of technical change is not significant. Norway is a major country in salmon farming. Terje and Helen (2011) utilized DEA 
Malmquist productivity index method to measure the efficiency of salmon farming in Norway. The results showed that the change of TFP was mainly caused by the change of technology, while the change of efficiency had little effect on it. It is also concluded that agglomeration externality is the main factor affecting salmon productivity and technical efficiency. Ji and Wang (2015) evaluated aquaculture efficiency of 16 provinces in China with SBM-DEA model, and they found that technology expansion and improvement of technology level have obvious positive effects on aquaculture. Pipitone and Colloca (2018) used Malmquist index method to decompose TFP of Italian trawl fishery. The results showed that overfishing had a negative effect on fishery efficiency, and capital accumulation and pricing strategy had a positive effect on fishery efficiency. $\mathrm{Xu}$ (2020) measured the TFP of mariculture in nine coastal provinces of China based on DEA Malmquist method, and pointed out that the growth of mariculture efficiency belongs to technology induced growth.

From the above studies, it can be seen that most of the existing research on fishery efficiency focuses on single species breeding, fishing, mariculture and overall fishery production efficiency, while few on the freshwater aquaculture efficiency. Moreover, no studies have considered the importance of regional heterogeneity for freshwater aquaculture efficiency by the meta-frontier. In terms of research methods, SFA function method, traditional DEA method and traditional Malmquist index method are mostly used. As for the analysis of the results, most of the existing studies are directly based on a frontier to analyze the overall situation. In order to make up for the defects of the existing research, this paper uses the meta-frontier method to measure the TFP of 
152

153

freshwater aquaculture in China based on the regional heterogeneity. The inherent assumption of meta-frontier method is that the technological progress of different groups is heterogeneous, so DMUs should be divided into different groups. Previous studies have suggested that geographical proximity and self-characteristics are the decisive factors for grouping (Oh and Lee, 2010). Considering the characteristics of freshwater aquaculture, this paper divides the areas into coastal regions and inland regions, which follows this principle.

In summary, there are three innovations in this paper. First, in terms of data and sample selection, this paper selects the input-output panel data of freshwater aquaculture in 11 coastal provinces and 17 inland provinces in China from 2003 to 2019. Compared with previous studies, this study has a larger time span and a wider area, which can more accurately reflect the contemporary freshwater aquaculture TFP in China. Second, in terms of research method, considering the regional heterogeneity, this paper uses DDF-MMI model to analyze the TFP of freshwater aquaculture in coastal and inland regions through the comparison of meta-frontier and group-frontier. Third, in the analysis of the results, this paper analyzes the TFP under the two conditions of overall and cluster group, and discusses the freshwater aquaculture technology gap rate (TGR) in coastal and inland regions.

\section{Methodology}

\subsection{DDF}

DDF was proposed by Chung et al. (1997) proposed to measure the production efficiency. DDF can actually reflect the economic effect (Yang et al., 2020), which is 
174

175

suitable for the measurement of freshwater aquaculture efficiency. This paper takes provinces as DMUs. Assuming that the number of DMUs is K, and the number of inputs and outputs for each DMU is $\mathrm{M}$ and $\mathrm{N}$, respectively, then the production possibility set of the current period can be expressed as:

$P^{t}\left(x^{t}\right)=\left\{y^{t} \mid x^{t}\right.$ can produce $\left.y^{t}\right\}, t=1,2,3 \ldots, T$

The definition of output oriented DDF is as follows:

$\vec{D}(x, y ; g)=\max \{\beta \mid y+\beta g \in P(x)\}$

Where $\mathrm{x}$ is the input vector and $\mathrm{y}$ is the output of the production. $\mathrm{g}$ is the direction vector, where is the output expansion direction, denoted by $(0, y) . \beta$ is the the value of DDF and represents the ratio of output expansion.

The specific process of using DDF to evaluate freshwater aquaculture efficiency is as follows:

$\operatorname{Max} \vec{D}(x, y ; g)=\beta$

s.t. $\left\{\begin{array}{l}\sum_{k=1}^{K} \lambda_{k} x_{k} \leq x \\ \sum_{k=1}^{K} \lambda_{k} y_{k} \geq(1+\beta) y \\ \lambda_{k} \geq 0, k=1,2, \ldots \ldots, K\end{array}\right.$ The closer each DMU is to the meta-front curve, the greater the efficiency of DMU is. The efficiency index of DMU is $1 /(1+\beta)$.

\subsection{The double-hierarchy meta-frontier DDF-DEA model}

Hayami and Ruttan(1971) proposed the concept of meta-frontier when considering heterogeneity. This method has been widely used in efficiency measurement after development (O'Donnell et al., 2008). On the basis of DDF, this paper adds regional 


$$
\operatorname{Max} \overrightarrow{D^{M}}\left(x_{m o}^{t}, y_{n o}^{t} ; g\right)=\beta^{M}
$$

$$
\text { s.t. }\left\{\begin{array}{c}
\sum_{t=1}^{T} \sum_{k=1}^{K_{M}} \mu_{k}^{t} x_{m, k}^{t} \leq x_{m o, k}^{t}, m=1,2, \ldots, M \\
\quad \sum_{t=1}^{T} \sum_{k=1}^{K_{M}} \mu_{k}^{t} y_{n, k}^{t} \geq\left(1+\beta^{M}\right) y_{n o, k}^{t}, n=1,2, \ldots, N \\
\mu_{k}^{t} \geq 0, k=1,2, \ldots, K_{M} ; t=1,2, \ldots, T
\end{array}\right.
$$
meta-frontier, $K_{M}=28$.

$$
\operatorname{Max} \overrightarrow{D^{R}}\left(x_{m o}^{t}, y_{n o}^{t} ; g\right)=\beta^{R}
$$

factors to the model by considering the meta-frontier and regional frontier. The regional frontier includes coastal regional frontier and inland regional frontier. As shown in Fig.1, there are two regional fronts under the envelope of the meta-frontier.

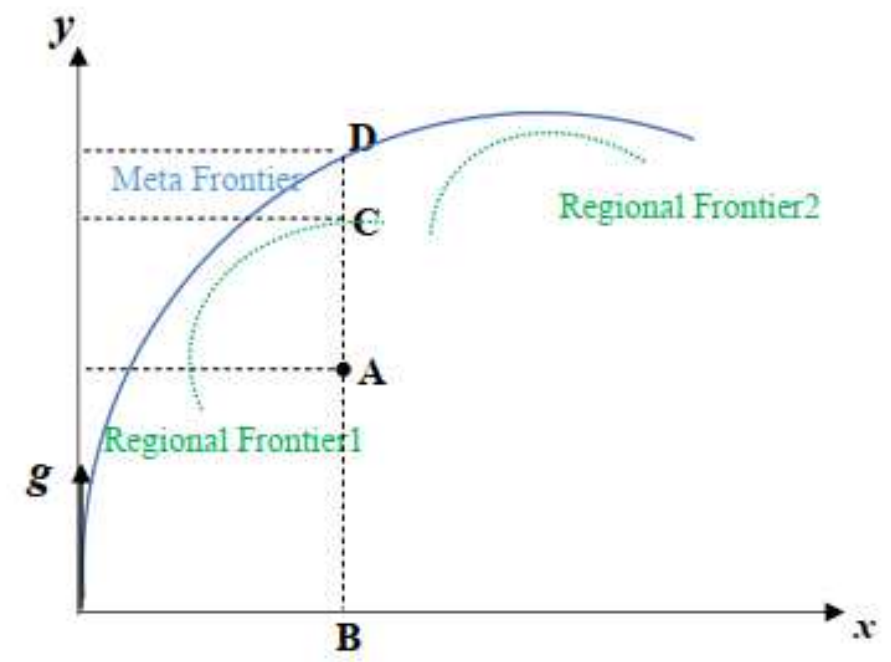

Fig.1. Double-hierarchy meta-frontier

Where $\beta^{M}$ is the proportion of output expansion under the meta-frontier. $\mathrm{M}, \mathrm{N}$ and $\mathrm{T}$ are the number of inputs, the number of outputs, and the number of periods respectively. $\mu$ is the intensity variable under the meta-frontier. $K_{M}$ is the number of DMUs in the

Second, the DDF function model at the regional frontier is shown in Eq.(5). 
s.t. $\left\{\begin{array}{c}\sum_{t=1}^{T} \sum_{k=1}^{K_{R}} \theta_{k}^{t} x_{m, k}^{t} \leq x_{m o, k}^{t}, m=1,2, \ldots, M \\ \quad \sum_{t=1}^{T} \sum_{k=1}^{K_{R}} \theta_{k}^{t} y_{n, k}^{t} \geq\left(1+\beta^{R}\right) y_{n o, k}^{t}, n=1,2, \ldots, N \\ \theta_{k}^{t} \geq 0, k=1,2, \ldots, K_{R} ; t=1,2, \ldots, T\end{array}\right.$

Where $\beta^{R}$ is the proportion of output expansion under the regional frontier. $\theta$ is the intensity variable under the regional frontier. $K_{R}$ is the number of DMUs in the regional frontier. In coastal region, $K_{R}=11$, and in the inland region, $K_{R}=17$.

\subsection{The double-hierarchy meta-frontier Malmquist index} proposed to apply Malmquist index (MI) method to measure TFP. The concept of MI was first proposed by Malmquist (1953) to analyze the cost change in different periods.

However, the traditional MI is based on the relative change of production frontier in the calculation process, rather than the absolute technology change (Zhong et al., 2020). In addition, the traditional MI has some limitations, such as no feasible solution, lack of transitivity and technical regression (Pastor and Lovell, 2005). Oh and Lee (2010) applied the method of meta-frontier to the global reference Malmquist model, constructed the meta-frontier Malmquist index (MMI) model, and further decomposed

it. This method includes all the evaluated DMUs in the global reference set, which overcomes the shortcomings of the traditional MI. MMI can measure TFP more accurately. The global production possibilities can be expressed as Eq.(6).

Where, $P^{t}\left(\mathrm{x}^{t}\right)=\left\{\mathrm{y}^{t} \mid \mathrm{x}^{t}\right.$ can produce $\left.\mathrm{y}^{t}\right\}, t=1,2,3 \ldots, T$ 
$229=\sqrt{\frac{1-\overrightarrow{D_{t-1}^{M}}\left(x^{t}, y^{t} ; g\right)}{1-\overrightarrow{D_{t-1}^{M}}\left(x^{t-1}, y^{t-1} ; g\right)} \times \frac{1-\overrightarrow{D_{t}^{M}}\left(x^{t}, y^{t} ; g\right)}{1-\overrightarrow{D_{t}^{M}}\left(x^{t-1}, y^{t-1} ; g\right)}}$

$231=\sqrt{\frac{1-\overrightarrow{D_{t-1}^{R}}\left(x^{t}, y^{t} ; g\right)}{1-\overrightarrow{D_{t-1}^{R}}\left(x^{t-1}, y^{t-1} ; g\right)} \times \frac{1-\overrightarrow{D_{t}^{R}}\left(x^{t}, y^{t} ; g\right)}{1-\overrightarrow{D_{t}^{R}}\left(x^{t-1}, y^{t-1} ; g\right)}}$

$232 M M I_{t-1, t}^{M}, M M I_{t-1, t}^{R}$ represents the TFP of freshwater aquaculture under the meta-

233 frontier and regional frontier respectively. If the value of MMI is greater than 1, it 234 indicates that the productivity increases from the previous period to this period. MMI 235 can be further decomposed into EC and TC, its decomposition is shown in Eq.(10):

$M M I_{t-1, t}=\sqrt{\frac{1-\overrightarrow{D_{t-1}}\left(x^{t}, y^{t} ; g\right)}{1-\overrightarrow{D_{t-1}}\left(x^{t-1}, y^{t-1} ; g\right)} \times \frac{1-\overrightarrow{D_{t}}\left(x^{t}, y^{t} ; g\right)}{1-\overrightarrow{D_{t}}\left(x^{t-1}, y^{t-1} ; g\right)}}$

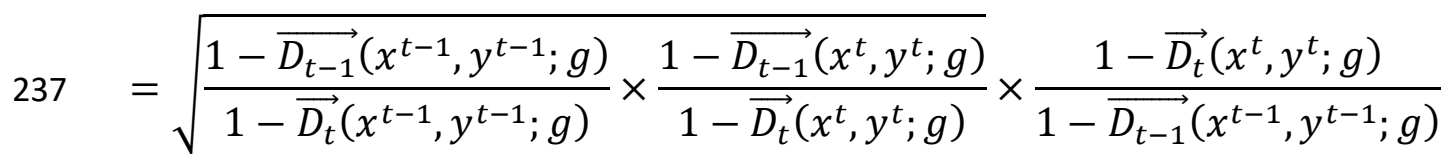

$238=T C_{t-1, t} \times E C_{t-1, t}$

A key index of meta-frontier method is technology gap ratio (TGR), which is the the gap between the actual technology level and the potential optimal technology level can be expressed as: $T G R_{t}=(\mathrm{BA} / \mathrm{BD}) /(\mathrm{BA} / \mathrm{BC})=\mathrm{BC} / \mathrm{BD}$. The value range is $[0,1]$, technology level. 


\subsection{Variables and sources of data}

The accuracy of DEA in measuring TFP is closely related to the choice of inputoutput variables. According to the viewpoint of western economics, the choice of input variables should combine land, capital and labor (Mankiw, 1997). Combined with the existing literature (Asche et al., 2013; Ji et al, 2015), this paper selects three variables to build an input variable system, which are freshwater aquaculture area, freshwater aquaculture professionals and freshwater seedling quantity. These three variables reflect land, labor and capital respectively. As for the selection of output variables, it is usually to use gross economic value of fishery to reflect the total scale and total results of fishery production in a period of time at fishery application (Zheng et al., 2019). Based on the actual situation of freshwater aquaculture, the output variable of this paper is the fishery production value of freshwater aquaculture. The specific explanation is as follows:

(1) Freshwater aquaculture area. The water surface area of aquaculture products in freshwater areas, including pond culture area, lake culture area, river aquaculture area and reservoir aquaculture area, etc. The unit of measurement is hectare.

(2) Freshwater aquaculture professionals. Fishery practitioners who have been engaged in freshwater aquaculture for more than six months or more than $50 \%$ of their livelihood sources depend on freshwater fishery activities throughout the year. Compared with the part-time and temporary employees of freshwater aquaculture, the professionals from industry are more representative. The unit of measurement is person. 
268

269

270

271

272

273

274

275

276

277 impact of inflation. The relevant data of input-output variables are all from "China

(4) The fishery production value of freshwater aquaculture. It refers to the output and outcomes of freshwater aquaculture fishery activities in the accounting period in the form of currency. The unit of measurement is 10000 yuan.

The input-output variable system is listed in Table 2.

The variables expressed in currency are bound to be affected by inflation, which will lead to the lack of objectivity and accuracy of data. For this reason, this paper selects 2003 as the base year to deal with the output variables, so as to eliminate the
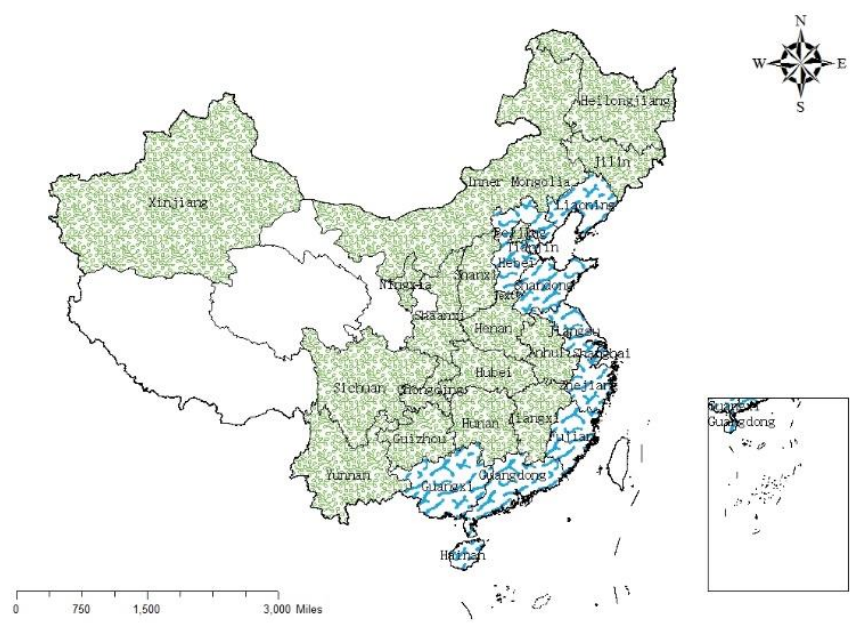

Fisheries statistical yearbook".

\section{5 study area}

Fig.2. Spatial distribution sampling in freshwater aquaculture areas

The paper aims to study freshwater aquaculture efficiency in China. The relevant 
and Qinghai provinces and cities is small, which is lack of research value. For these reasons, this work selects 28 provinces with more freshwater aquaculture, such as Fujian, Guangdong, Anhui and Beijing, as samples. As shown in Fig.2, the areas involved are divided into coastal and inland regions.

\section{Empirical analysis}

This work uses MaxDEA software to calculate the TFP and its decomposition index values of freshwater aquaculture in 11 coastal provinces and 17 inland provinces from 2004 to 2019. According to the calculation results, this part analyzes the development trend and regional characteristics of China's freshwater aquaculture.

\subsection{Temporal and spatial characteristics of TFP in freshwater aquaculture}

The overall fluctuation trend of TFP, EC and TC in China's freshwater aquaculture is basically the same at the meta-frontier and group frontier (Fig.3). The average annual TFP of freshwater aquaculture basically reached more than 1 both at the meta-frontier and group frontier, except in 2009 and 2011. From 2004 to 2013, TFP fluctuated greatly. After 2013, TFP is relatively stable. Specifically, TFP was the lowest in 2011 and the highest in 2012. Between these two years, TFP fluctuated the most, with meta-frontier of 0.811 and group frontier of 0.827 . This is mainly because in 2012, the central government attached great importance to the development of Fisheries and gave the highest amount of funding in the history of Fisheries Finance. According to statistics, the total investment in fishery infrastructure construction in the whole year reached 8.933 billion yuan, nine times that of 2011. Driven by the strong central investment, the level of fishery infrastructure has been significantly improved. In addition, the 
technological system of modern fishery industry was further improved in 2012. Seven key fishery technology research projects have been included in the national public welfare agricultural industry research special plan, with funding support of 106 million yuan. 184 patents were invented in fishery in the whole year, with a year-on-year increase of $142.11 \%$. At the same time, the promotion of aquatic technology has been steadily promoted. In 2012, a total of 2.9534 million fishermen were trained, which effectively promoted the improvement of fishermen's quality and the popularization of aquaculture technology and management technology. From 2012 to 2013, TFP fell the most, with the meta-frontier of 0.617 and the group frontier of 0.619 . From 2013 to 2019, the trend of TFP is moderate. It shows that the development of China's freshwater aquaculture has begun to stabilize after 2013. This may be caused by the following reasons: First of all, during the "Eleventh Five Year Plan" period, the national fishery system adheres to the guidance of the scientific development and solidly promotes the construction of modern fishery. This makes the structure of China's fishery industry further optimized, and the level of fishery development and industrial competitiveness significantly improved; then, during the "Twelfth Five Year Plan" period of fishery development, fishery is facing the following favorable conditions: First, China has begun to improve the long-term mechanism of promoting agriculture through industry and leading rural areas by cities. It is in the critical period of speeding up the transformation of traditional agriculture and taking the road of agricultural modernization with Chinese characteristics. In this way, the freshwater aquaculture industry is promoted in an all-round way. Second, after long-term development, fishery 
has formed a large industrial scale and a good industrial foundation. And fishery has accumulated rich experience in production, management and technology. At the beginning of the "Thirteenth Five Year Plan", the industrial structure of China's freshwater aquaculture has been further adjusted. The input of resource elements, technical level and management level have developed to a relatively saturated level. These factors make the input-output conversion rate of freshwater aquaculture develop more smoothly. However, it could not be ignored that the degree of scale, intensification and organization of China's fishery production is still low on the whole. The quality of aquaculture practitioners is also low. These factors will restrict the sustainable development of freshwater aquaculture industry, and then make the efficiency of freshwater aquaculture at an effective but not high level. As shown in Fig.3, the average annual TFP in 2013-2019 is closely around 1. Taking the year with the highest annual TFP as an example, in 2017, the average annual TFP under the meta-frontier and group frontier was only 1.146 and 1.153 , respectively. At the meta-frontier, the provinces that do not reach the effective state of freshwater aquaculture are: Fujian (0.869), Hebei (0.999), Liaoning (0.699), Tianjin (0.906) in coastal region, Henan (0.980), Hubei (0.944), Shaanxi (0.589), Xinjiang (0.743) in inland region. At the group frontier, provinces with inefficient freshwater aquaculture are as follows: Fujian (0.869), Hebei (0.995), Liaoning (0.697), Tianjin (0.906) in coastal region, Shaanxi (0.636), Xinjiang (0.810) in inland region. The ineffectiveness of input and output of freshwater aquaculture in these provinces has caused great losses. 

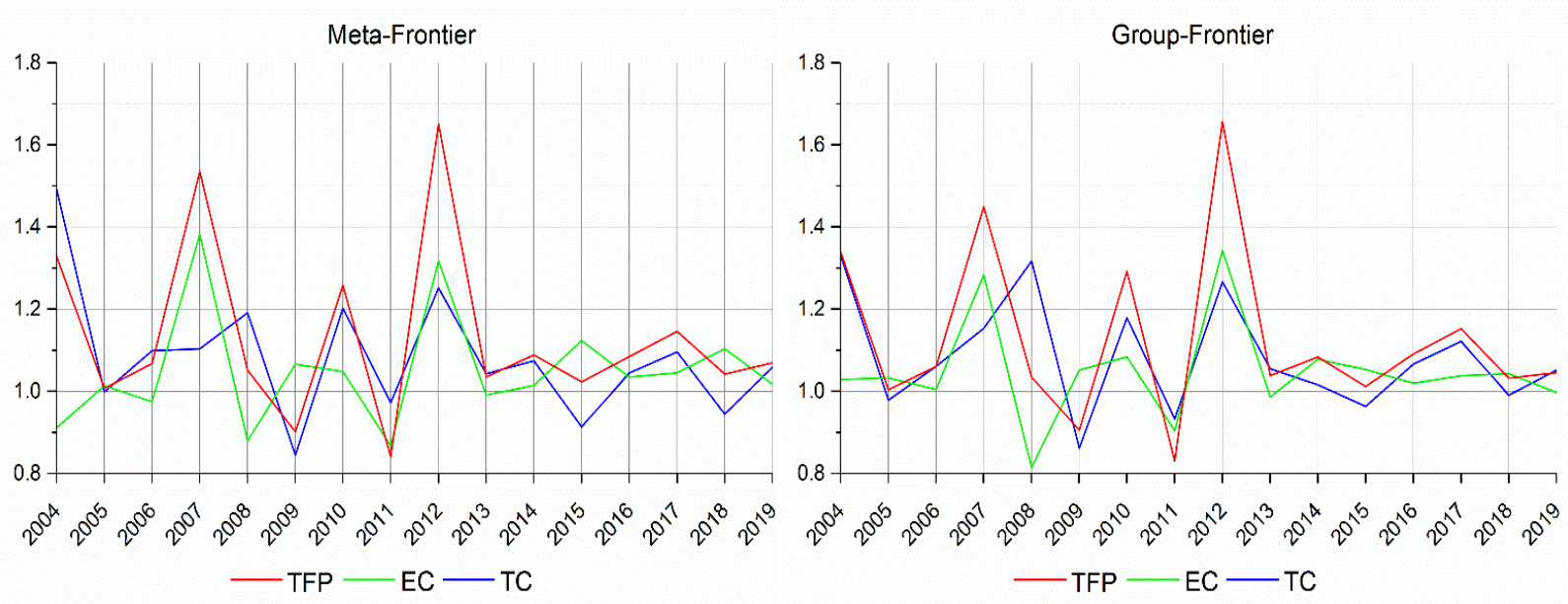

Fig.3. The trends of TFP、EC and TC at two frontiers

The changing trend of TFP is roughly the same as that of EC and TC both at meta-

350 frontier and group-frontier (Fig.3). This shows that the changes of TFP in China's

$53.6 \%$ and $45 \%$ compared to the previous year in meta-frontier and group frontier, 
severe fishery production situation, it is difficult for fishery production management to keep up in a short time. From 2013 to 2019, both EC and TC maintained a relatively stable level, and fluctuated closely around 1 slightly. This showed that after 2013, the management level and technical level of China's freshwater aquaculture were at a relatively stable level of development. In general, the development of freshwater aquaculture in China has management advantages. The technology level has also reached a stable and better level. However, there is still no breakthrough. Therefore, it is very necessary to cultivate professional management and technical personnel. At the same time, China should pay attention to the construction of freshwater aquaculture technology system, increase investment in fishery technology elements, continue to carry out technological innovation, and strive to achieve technological breakthroughs as soon as possible. This will effectively promote the improvement of freshwater aquaculture TFP and contribute to the sustainable development of freshwater aquaculture. 
( I ) Average of TFP of freshwater aquaculture in meta-frontier of China's 28 provinces in 2004-2019

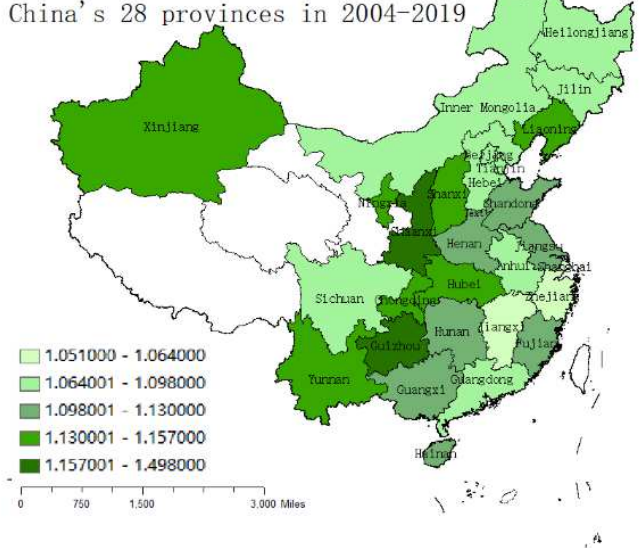

(III) Average of TFP of freshwater aquaculture in group-frontier of

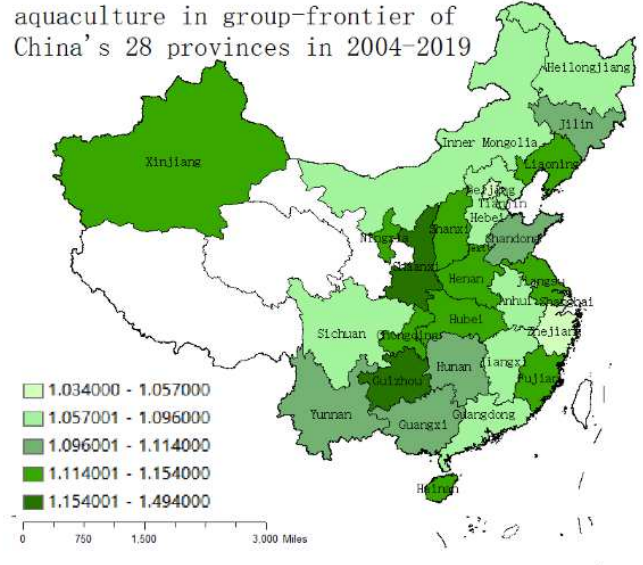

(II) Top and last five of Freshwater Aquaculture in Matefrontier

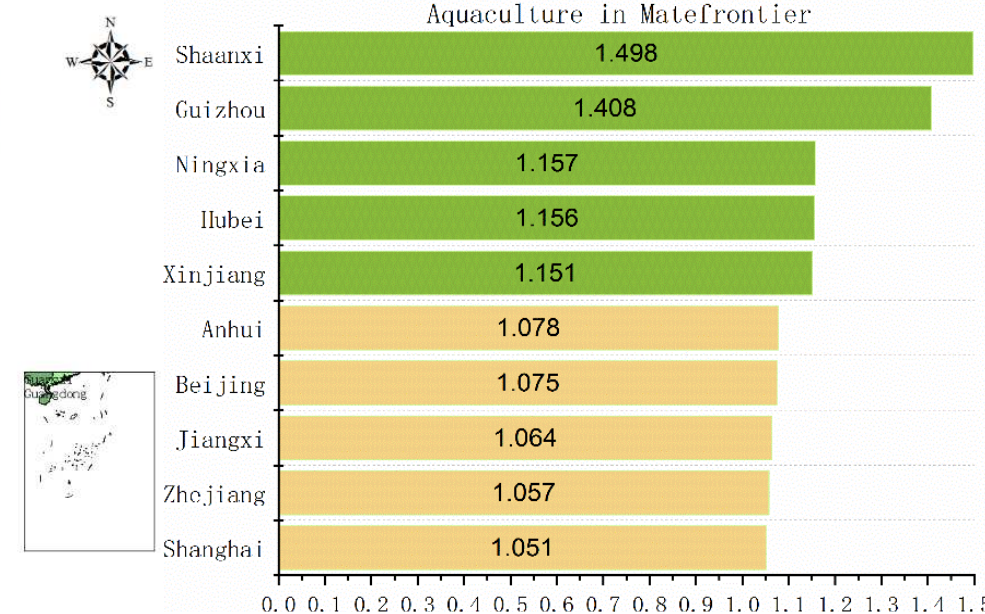

(IV) Top and last five of Freshwater

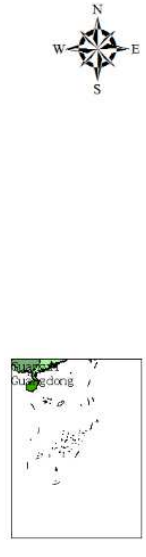

Aquaculture in Groupfrontier

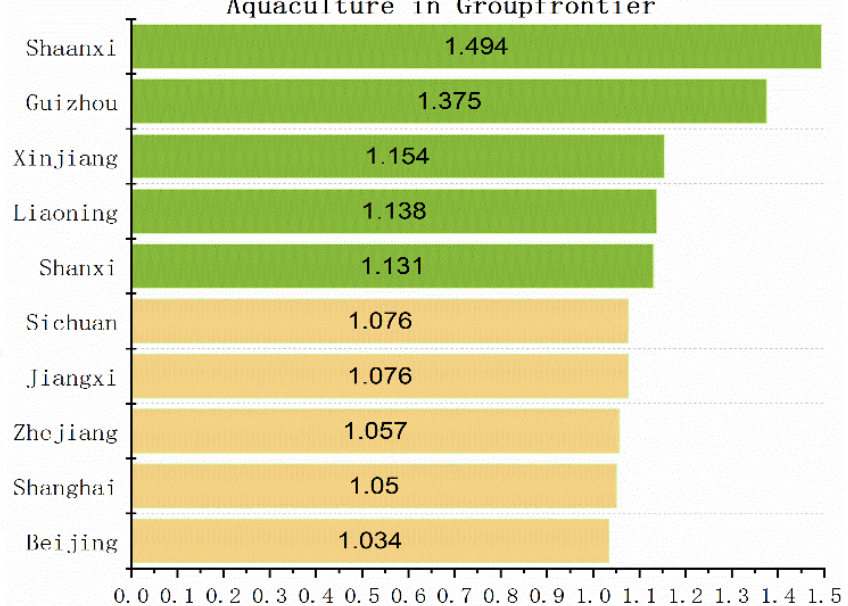

Fig.4. Averages of TFP of freshwater aquaculture in two frontiers 
scientific and technological investment in aquaculture and used scientific guidance to effectively prevent the development of diseases. On the other hand, Liaoning has a good level of aquaculture management. Liaoning has been committed to the establishment and improvement of aquaculture mechanisms, including inputs and aquaculture water testing. At the same time, with the continuous development of the level of technology and management, the scale of the aquaculture industry in Liaoning has also continued to expand. Shaanxi, Guizhou and Xinjiang are in the top five of TFP both at the two frontiers (Fig.4. ( II ), (IV)), and these three provinces are all inland cities. Among them, Shaanxi is in the first place. This is mainly because Shaanxi had a very high TFP in 2012, which was largely due to the strong investment and support of the central government for fishery infrastructure construction in 2012. At the same time, Shaanxi government have also implemented financial discounts to support fishery infrastructure. In addition, the fishery department of Shaanxi organizes fishery science and technology personnel to go to the grassroots level to promote aquaculture technology. It can be seen that technological progress and management level improvement are really critical for the sustainable development of freshwater aquaculture. Shanghai, Zhejiang, Jiangxi, and Beijing are all ranked in the bottom five under the two frontiers (Fig.4. (II), (IV)). Among them, Shanghai and Zhejiang are both coastal provinces.

As shown in Fig.5, the EC and TC of Shaanxi, Guizhou and Xinjiang in the two frontiers are really considerable, especially in Shaanxi and Guizhou. This determines that these provinces have higher TFP. As for the provinces with the lowest TFP, The values of TC in Shanghai, Zhejiang, Jiangxi and Beijing are not low, all greater than 1. 
411 progressive. The lower TFP of Shanghai, Zhejiang and Beijing is mainly due to the

412 lower EC value. The average EC Value of Shanghai, Zhejiang and Beijing is less than

413 1. This shows that the management level of Fisheries in these provinces is at a declining

414 level. This is mainly because, in recent years, Shanghai, Zhejiang, Beijing, Jiangxi pay

415 more and more attention to the development of electronic information industry and

416 modern service industry, and gradually develop into the main gathering place of China's

417 electronic information industry cluster and business exhibition cluster area, while

418 ignoring the management of fishery. It can be further found that if these provinces with

419 high technical level are willing to further improve their fishery management level, their

420 TFP of freshwater aquaculture will be greatly improved, which will help to promote the

421 sustainable development of freshwater aquaculture.

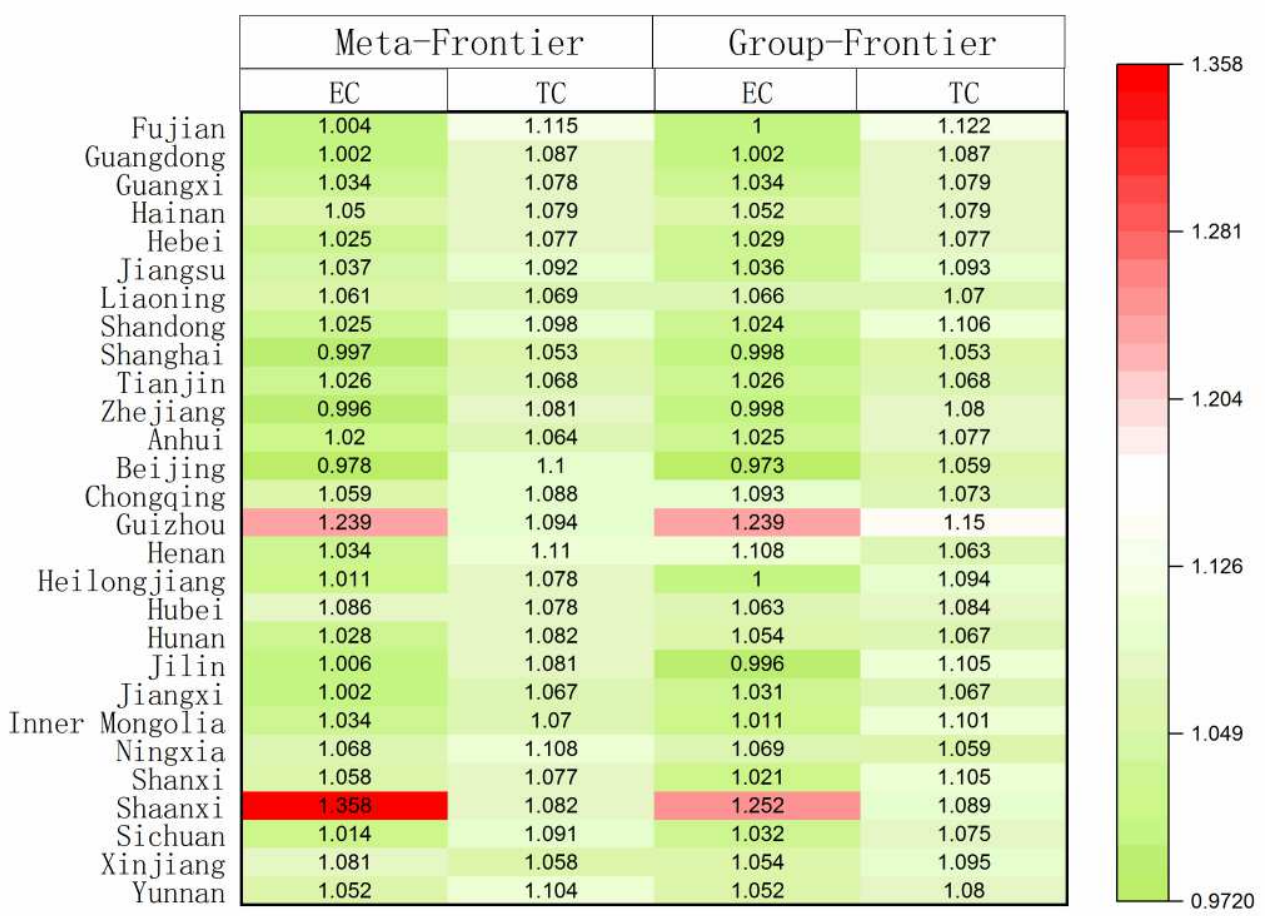

Fig.5. Averages of EC and TC of freshwater aquaculture in two frontiers 
means that the technology is in a state of progress. However, in the single frontier, more

425 than half of the provinces' TC values are below the average. In the meta-front and group

426 front, the provinces with TC values below the average are Anhui, Hunan and Jiangxi in

427 inland region, and 7 provinces in coastal region, including Guangxi, Hainan and Hebei

428 etc. This also shows that there is a large spatial differences in freshwater aquaculture

429 technology, and there is a large space for improvement of technology. Hence, it is

430 necessary to strengthen the exchange and cooperation of freshwater aquaculture

431 technology between provinces. Especially for provinces with low level of freshwater

432 aquaculture technology, the local government should actively introduce advanced

433 technology and strengthen the promotion of freshwater aquaculture technology.
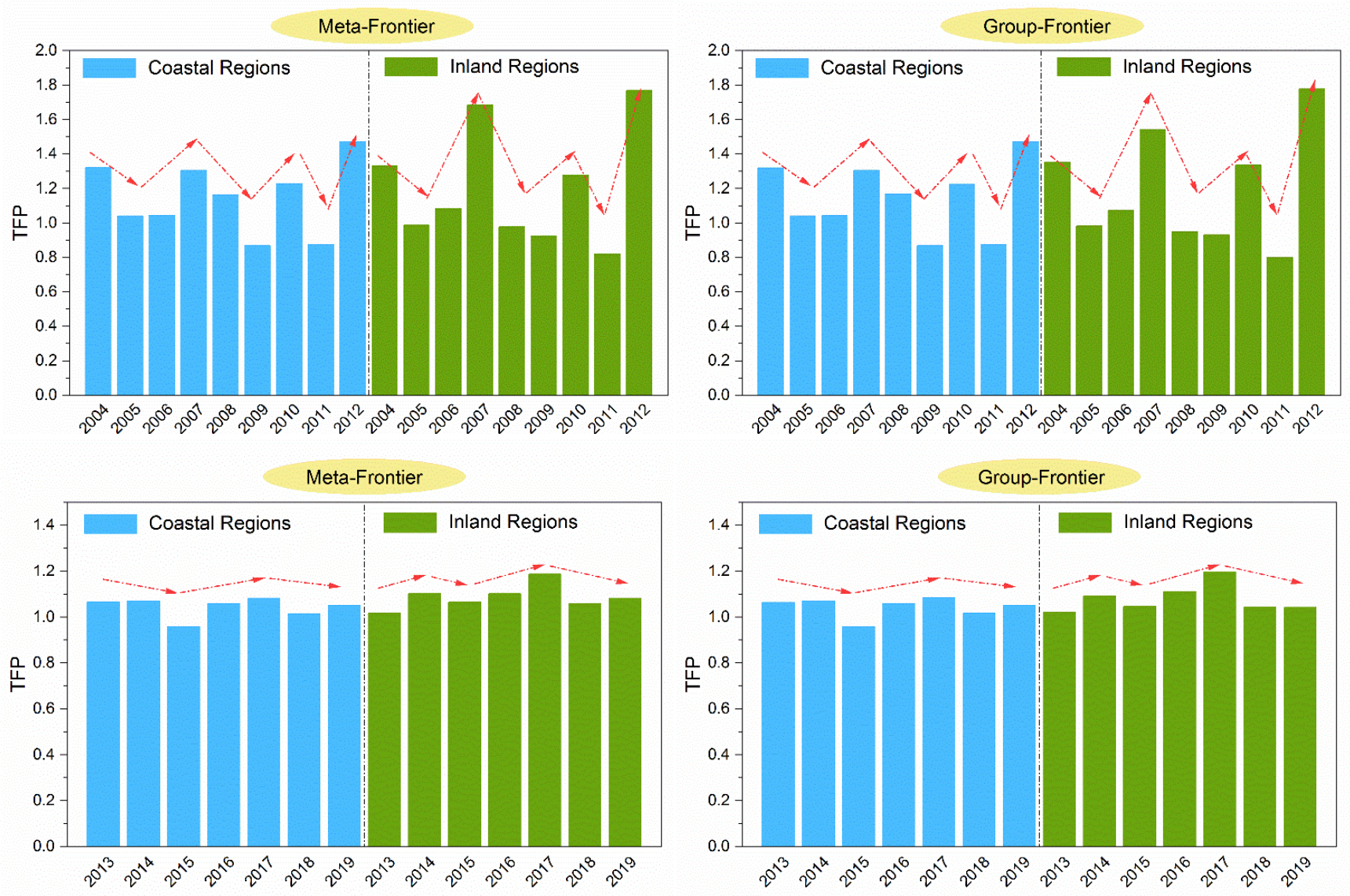
Fig.6. Average annual TFP of freshwater aquaculture in two regions

435

\subsection{Temporal and spatial characteristics of TFP in freshwater aquaculture}

\section{between regions}

It can be seen from Fig.6, whether it is in coastal or inland regions, freshwater aquaculture TFP fluctuated greatly during 2004 to 2012. In the coastal region, the TFP was the lowest in 2009, with 0.868 and 0.867 at the meta-front and the group front, respectively, representing a decrease of $13.2 \%$ and $13.3 \%$ from the previous year. TFP was the highest in 2012, with 1.472 for both frontiers, an increase of $47.2 \%$ over the previous year. As for the inland region, the lowest TFP was in 2011, and the metafrontier and group frontier were 0.819 and 0.800 respectively, they are decreased by $18.1 \%$ and $20 \%$ from the previous year. In the inland area, the lowest TFP was in 2011, and its values were 0.819 and 0.800 under the meta-frontier and group frontier, respectively, which were $18.1 \%$ and $20 \%$ lower than the previous year. The highest TFP was in 2012, which was 1.768 under the meta-frontier, an increase of $76.8 \%$ over the previous year, and 1.777 under the group frontier, an increase of $77.7 \%$ over the previous year. From 2012 to 2013, TFP showed a decline both at coastal and inland regions. After 2013, TFPs began to show steady and small fluctuations. It can be seen that after 2013, both coastal and inland regions have begun to enter a relatively stable level in the development of freshwater aquaculture. Moreover, after 2013, TFPs were greater than 1, which means freshwater aquaculture remained in an effective state, except in 2015. This also indicates that the development level of freshwater aquaculture in coastal and inland regions has been steadily improved after 2013. The freshwater 
457 structure has been further optimized. However, there has not been an amazing 458 breakthrough value. This is consistent with the analysis of the time characteristics of

459 the overall national TFP in Section 4.1.
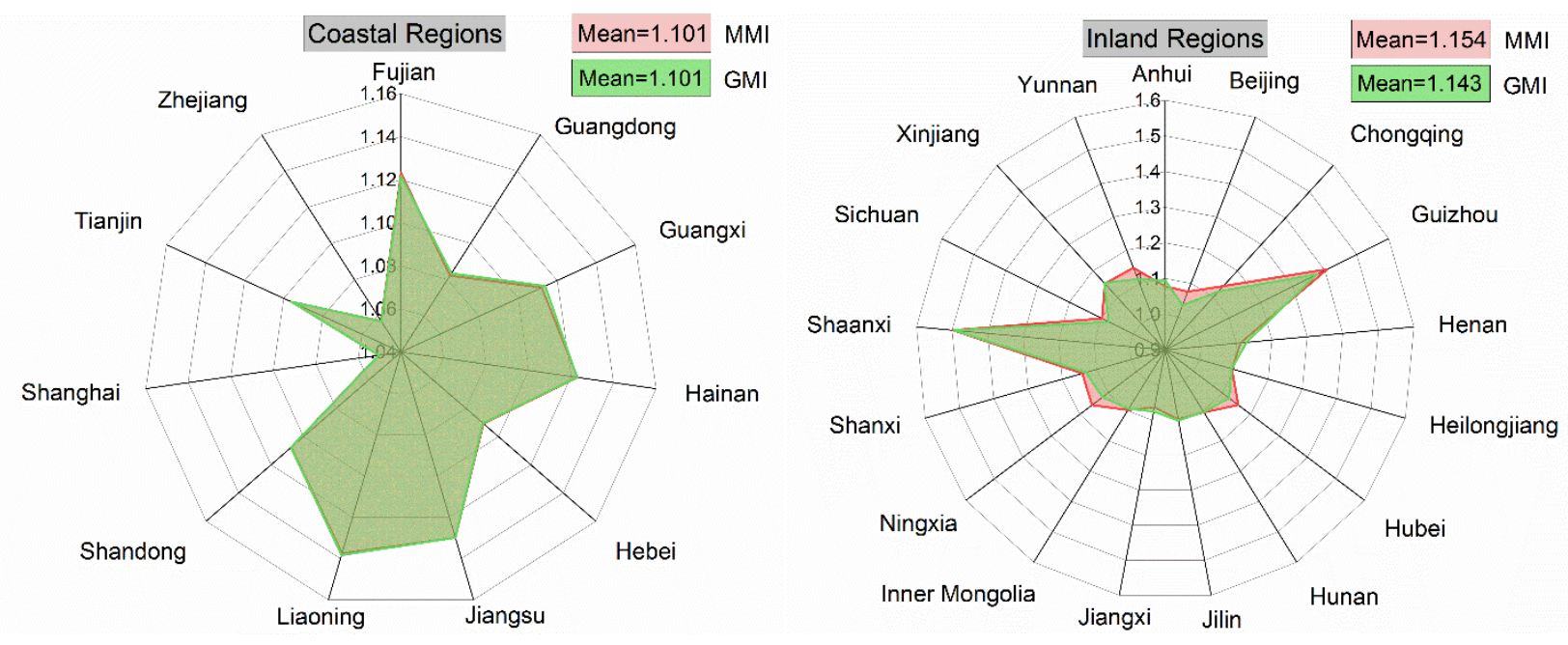

Fig.7. Provinces average TFP of freshwater aquaculture in two regions

meta-frontier were 1.101 and 1.154 respectively (Fig.7). It can be seen that under the meta-frontier, the freshwater aquaculture efficiency of coastal region is significantly lower than that of inland region. TFP (GMI) of coastal and inland at group frontier were 1.101 and 1.143 , respectively. That is to say, the freshwater aquaculture efficiency in inland region is still better than that in coastal region. It shows that the development of

467 freshwater aquaculture in inland region is more advantageous regardless of the regional 468 factors. Combined with Fig.8, the average values of TC and EC in coastal region are lower than those in inland region. Especially for EC, the average value of EC in coastal region is significantly lower than that in inland region. In addition, the EC values in coastal region are more concentrated, while those in inland areas are more dispersed. It 


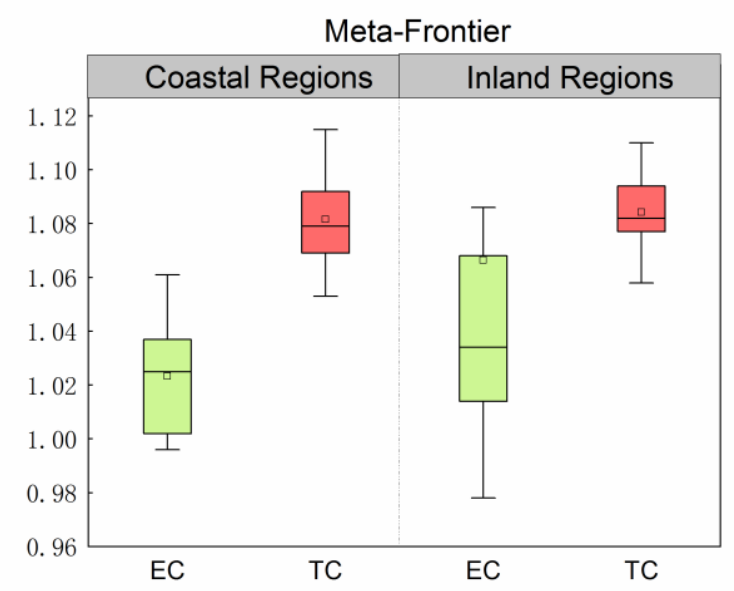

can be seen that there are great differences in the management level of freshwater aquaculture of inland provinces. This means that there is still much space for improvement in freshwater aquaculture management in many inland provinces.

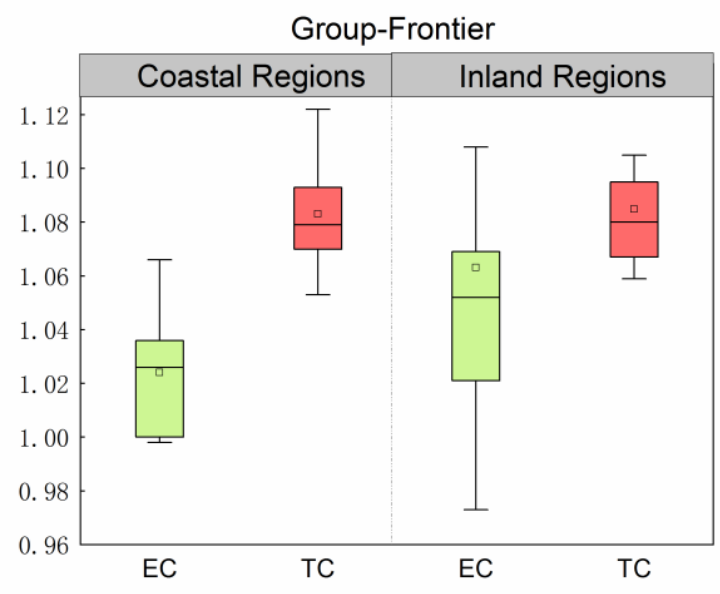

Fig.8. EC and TC of freshwater aquaculture in two regions

Coastal region has relatively developed economies and high levels of science and technology. With the development of society, they have increasingly concentrated on the development of high-tech industries and tertiary industries, thus they have ignored the development of agriculture to a certain extent. At the same time, coastal region are rich in seawater resources, and the emphasis on marine fisheries has made a lot of fishery production factors invested in marine fisheries, thus slowing the development of freshwater fisheries. However, many provinces in the inland region still focus on the primary industry. Taking Heilongjiang and Shanxi as examples, the primary industry in Heilongjiang accounts for the highest proportion of the three industries, and it is also showing a gradual upward trend. The emphasis on the primary industry has made its agriculture, including the freshwater fishery industry, develop on a larger scale. Therefore, the local freshwater aquaculture TFP is relatively high. As for Shanxi, although it is not a major fishery province, the province attaches great importance to 
the development of fisheries. In the past few years, Shanxi has been committed to the transformation of fishery production methods and improving the level of intensification of the fishery industry. In addition, advanced aquaculture technology has been introduced through the "Three North" technical cooperation group. And at the same time, the local area is also making efforts to promote freshwater aquaculture technology.

With the joint action of many parties, the technical ability of fish farming management in Shanxi has been greatly improved. In summary, the top priority for freshwater aquaculture in coastal region is to invest necessary management resources to increase their freshwater aquaculture management level. Some provinces with lower management levels in inland region should actively seek communication between provinces to coordinate the improvement of freshwater aquaculture management.

\subsection{Analysis of TGR in regional freshwater aquaculture}

The full name of TGR is technology gap rate, which reflects the technology gap between group frontier and meta-frontier. The higher the TGR value is, the closer the DMU's technical level is to the potential optimal level.

The average TGR of coastal and inland regions are 0.997 and 0.740 respectively (see Fig.9( I )). The results show that the technical level of freshwater aquaculture in coastal region has reached $99.7 \%$ of the meta-frontier level, while that in inland region has only reached $74 \%$. This means that the technical level of freshwater aquaculture in coastal region is more in line with the potential optimal technology level, while the technical efficiency in inland region has a large gap with the optimal level, and there is more space for improvement. From the TGR value of each province, the value of TGRs 
in the coastal region were concentrated in a really close level, which shows that the

512 freshwater aquaculture technology level of each place in the coastal region has

513 agglomeration. The TGRs of the provinces in the inland region were relatively scattered,

514 which means that the development of freshwater aquaculture technology in the inland

515 region was quite uneven. Hence, it is very necessary to strengthen the exchange and

cooperation in freshwater aquaculture technology between provinces in inland region.

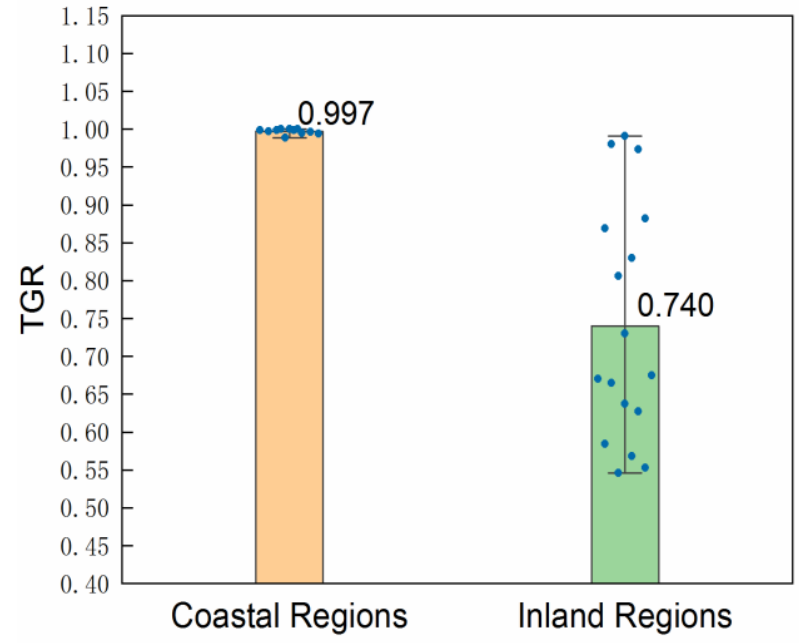

517

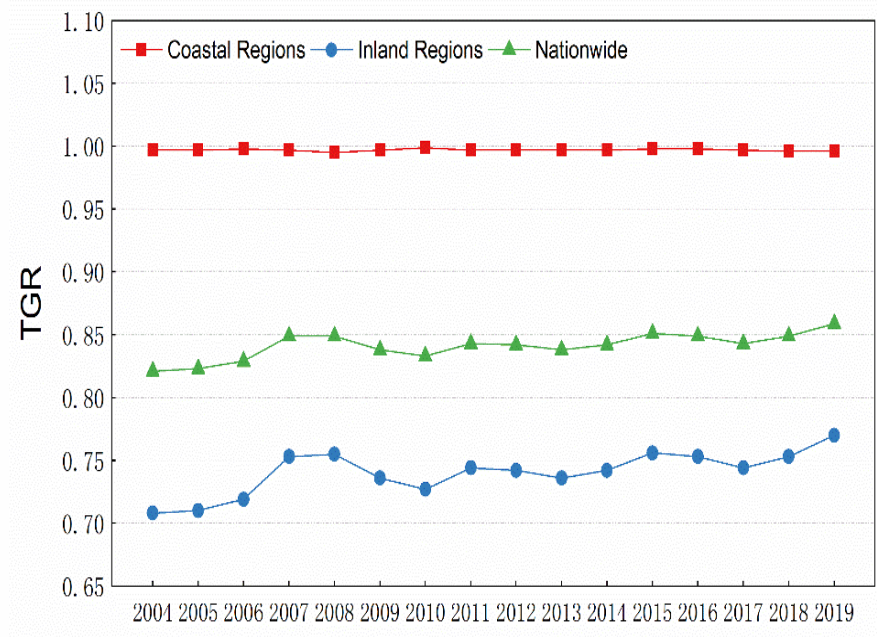

(II)

Fig.9. TGR of freshwater aquaculture

From a dynamic point of view (Fig. 9( II )), from 2004 to 2019, TGR in coastal region had been stable at around 1, with no major fluctuations. It also shows that the level of freshwater aquaculture technology in coastal region is very close to the optimal level of potential technology. On the whole, the TGR in the inland region showed a rising state from 2004 to 2019 , with the value from 0.708 to 0.77 . During the period from 2008 to 2010 , the TGR in the inland region experienced successive declines, which may be affected by the rising labor and resource costs. After 2010, TGR began to rebound and showed a relatively stable trend. In recent years, TGR in inland region 
has shown an upward trend. With the continuous introduction of advanced technologies in inland areas, its TGR is expected to maintain a growing trend.

Combining the previous section of the analysis of freshwater aquaculture TFP in coastal and inland regions, the overall performance of inland region is better than coastal region in the freshwater aquaculture industry. But when it comes to TGR, coastal region has absolute advantages. The main reason is that coastal areas have been the frontiers to take the lead in developing industrial clusters since reform and opening up in China. Then, through in-situ expansion, market pull, off-site incubation, and foreign transplantation and other ways, new technologies are continuously introduced and popularized, and development restrictions on the original location are broken. This has led to further advances in technology, so as to drive the development of the local economy. Obviously, there is a high correlation between economic development and technological development. Thus, the development of economy further promotes the progress of science and technology, and the development of economy and science and technology form a complementary effect. For these reasons, the technical efficiency level of freshwater aquaculture in coastal region is relatively high. Coastal region should give full play to their technological advantages, invest necessary elements in freshwater aquaculture, and continuously optimize the fishery industry. As for inland region, the overall economic development is not as good as that of the coastal region. In contrast, the aquaculture technology level is lagging behind, and it is more inclined to traditional aquaculture methods in terms of fish farming. Therefore, in addition to giving full play to their own resource advantages, inland region should also devote 
549

550

551

552

553

554

555

556

557

558

559

560

561

562

563

564

565

566

567

568

569

570

themselves to the improvement of freshwater aquaculture technology, and constantly approach the potential optimal technical level. Thereby promoting the sustainable development of the freshwater fishery industry.

\section{Conclusions and policy recommendations}

This paper constructs a double-hierarchy meta-frontier Malmquist index based on the DDF-DEA model to calculate the freshwater aquaculture TFP of 28 provinces in China from 2004 to 2019, and draws the following conclusions through analysis:

(1) TFP of freshwater aquaculture shows dynamic fluctuations and spatial differences both at meta-frontier and group frontier. From the perspective of time, TFP fluctuated sharply, especially from 2004 to 2013. After 2013, TFP showed slight and stable fluctuations between years. From a spatial point of view, combined with regional heterogeneity, inland region has obvious advantages in freshwater aquaculture efficiency compared with coastal region.

(2) According to the calculation of the decomposition index of TFP, the change of TFP in freshwater aquaculture is driven by the combined effect of EC and TC. In terms of regions, the relatively low TFP in coastal region is largely due to the low value of EC. It means that coastal regions are not making efforts to improve freshwater aquaculture management. The overall situation in the inland region is better than that in the coastal region, but the spatial differences of EC in the inland region are greater. Therefore, it can be seen that there is still a lot of space for development in freshwater aquaculture management capabilities in both coastal and inland regions. It is an entry point to promote the sustainable development of freshwater 
aquaculture.

572 (3) The TGR of freshwater aquaculture in coastal region has always been in a leading 573 position, with the value maintained at around 1, which is quite close to the potential aquaculture to a certain extent.

Based on the above conclusions, this paper proposes the following suggestions to improve the efficiency of freshwater aquaculture and promote the sustainable development of freshwater aquaculture industry.

(1) Strengthen the construction of aquatic science and technology system, and form a long-term mechanism for transforming technological achievements of freshwater aquaculture into actual productivity. Technical level plays a very important role in the freshwater aquaculture process. However, both the TC value and the TGR show an imbalance in space. Many provinces in China have greater space for improvement in freshwater aquaculture technology. Especially in inland region, the TGR is significantly lower than that in coastal region. Therefore, the government should increase the R\&D of freshwater aquaculture, and implement the research results into the breeding process, thus to increase the conversion rate of aquatic science and technology achievements, and promote the development of freshwater fishery technology industrialization. Especially in areas with low freshwater aquaculture technology, more attention should be paid to the improvement of 

industry, and actively introduce advanced technologies to improve the freshwater aquaculture production efficiency and promote its sustainable development.

(2) Improve the management level of freshwater aquaculture. The management level of freshwater aquaculture in coastal region is generally lower than that of inland region. Hence, on the basis of its technical advantages, coastal region should vigorously cultivate management talents and strengthen the improvement of freshwater aquaculture management capabilities. As for inland region, there are obvious spatial differences in the degree of change in technical efficiency. Therefore, inland region should improve the coordination of management capabilities between provinces according to their own management level, so as to increase the contribution of management capabilities to the freshwater aquaculture efficiency.

(3) Coordinate the differences between regions and balance the spatial layout of the freshwater aquaculture industry. Based on the considerable spatial differences of freshwater aquaculture in China, regional resource endowments, economic development level and technological level should be fully considered to form an interactive cooperation mechanism between coastal region and coastal region, coastal region and inland region, inland region and inland region. Thus to enable each region to play its own comparative advantage. This will ensure that advanced freshwater aquaculture technology and management methods can be effectively promoted and popularized. So that the efficiency of freshwater aquaculture in each area can be improved, and a spatial pattern of coordinated development will be 

freshwater aquaculture.

617

618

619

620

621

622

623

624

625

626

627

628

629

630

631

632

633

634

635

\section{Funding}

This work was supported by the National Social Science Foundation Project of

China (No. 18JL094).

\section{Authors' contributions}

Shen Zhong: Conceptualization, Methodology, Software. Aizhi Li: Investigation, Data curation, Formal analysis, Writing-Original draft. Jing Wu: Validation, WritingReviewing and Editing.

\section{Acknowledgements}

We thank the funding supported by National Social Science Foundation of China (No. 18JL094). And the authors are also grateful to anonymous referees for providing helpful comments and suggestions to improve this study.

\section{References}

Aigner D, Lovell CAK, Schmidt P (1977) Formulation and estimation of stochastic frontier production function models. Journal of Econometrics 6(1):21-37. https://doi.org/10.1016/0304-4076(77)90052-5

Asche F, Guttormsen AG, Nielsen R (2013) Future challenges for the maturing Norwegian salmon aquaculture industry: An analysis of total factor productivity change from 1996 to 2008. Aquaculture 396-399:43-50. 
636

637

Battese GE, Coelli TJ (1995) A model for technical inefficiency effects in a stochastic frontier production function for panel data. Empirical Economics 20(2):325-332. https://doi.org/10.1007/BF01205442

Charnes A, Cooper WW, Rhodes E (1979) Measuring the efficiency of decision making units. European Journal of Operational Research 2(6):429-444. https://doi.org/10.1016/0377-2217(78)90138-8

Chung YH, Färe R, Grosskopf S (1997) Productivity and Undesirable Outputs: A Directional Distance Function Approach. Journal of Environmental Management 51(3):229-240. https://doi.org/10.1006/jema.1997.0146

Coelli T (1998) A multi-stage methodology for the solution of orientated DEA models. Operations Research Letters 23(3):143-149. https://doi.org/10.1016/S0167$\underline{6377(98) 00036-4}$

FAO (2018) The State of World Fisheries and Aquaculture. Rome, Italy.

Färe R, Grosskopf G, Lovell CAK, Pasurka C (1989) Multilateral Productivity Comparisons When Some Outputs are Undesirable: A Nonparametric Approach. The Review of Economics and Statistics 71(1): 90-98. https://doi.org/10.2307/1928055

Färe R, Grosskopf S, Norris M, Zhang ZY (1994) Productivity growth, technical progress, and efficiency change in industrialized countries. American Economic Review 84(1):66-83.

Hassanpour B, Ismail MM, Mohamed Z, Kamarulzaman NH (2011) Factors affecting technical change of productivity growth in rainbow trout aquaculture in Iran. 
African Journal of Agricultural Research 6(10):2260-2272.

Hayami Y, Ruttan VW (1971) Agricultural Development: An International Perspective. Baltimore, London.

Iinuma M., Sharma KR, Leung P (1999) Technical efficiency of carp pond culture in peninsula Malaysia: an application of stochastic production frontier and technical inefficiency model. Aquaculture 175(3):199-213. https://doi.org/10.1016/S00448486(99)00033-2

Jamnia AR, Mazloumzadeh SM, Keikha AA (2015) Estimate the technical efficiency of fishing vessels operating in Chabahar region, Southern Iran. Journal of the Saudi Society of Agricultural Sciences 14(1):26-32. https://doi.org/10.1016/j.jssas.2013.04.005

Ji JY, Wang PP (2015) Research on China's aquaculture efficiency evaluation and influencing factors with undesirable outputs. Journal of Ocean University of China 14(3):569-574. https://doi.org/10.1007/s11802-015-2679-9

Kim DH, Lee KH, Bae BS, Park SW (2011) Productive efficiency of the sandfish Arctoscopus japonicus coastal gillnet fishery using stochastic frontier analysis. Fisheries Science 77(1):35-40. https://doi.org/10.1007/s12562-010-0308-5.

Kumbhakar SC, Lovell CAK (2000) Stochastic frontier analysis. Cambridge, U.K.

Lin BQ, Ge JM (2019) Carbon sinks and output of China's forestry sector: An ecological economic development perspective. Science of the Total Environment 655:1169-1180. https://doi.org/10.1016/j.scitotenv.2018.11.219

Malmquist S (1953) Index Numbers and Indifference Surfaces. Trabajos de Estadistica 
681

682

683

684

685

686

687

688

689

690

691

692

693

694

695

696

697

698

699

700

701

Mankiw NG (1997) Principles of economics. London: Oxford University Press.

Meeuseen W, Van den Broeck J (1977) Efficiency measurement from CobbDouglass production functions with composed error. International Economic Review 18:435-444. https://doi.org/10.2307/2525757

Merino G, Barange M, Blanchard JL, Harle J, Holmes R, Allen I, Allison EH, Badjeck MC, Dulvy NK, Holt J, Jennings S, Mullon C, Rodwell LD (2012) Can marine fisheries and aquaculture meet fish demand from a growing human population in a changing climate? Global Environmental Change 22(4):795-806. https://doi.org/10.1016/j.gloenvcha.2012.03.003

Muir J (2005) Managing to harvest? Perspectives on the potential of aquaculture. Philosophical Transactions. Biological Sciences 360(1453):191-218. https://doi.org/10.1098/rstb.2004.1572

O'Donnell C, Rao D, Battese G (2008) Metafrontier frameworks for the study of firmlevel efficiencies and technology ratios. Empirical Economics 34(2):231-255. https://doi.org/10.1007/s00181-007-0119-4

Oh DH, Lee JD (2010) A metafrontier approach for measuring Malmquist productivity index. Empirical Economics 38(1):47-64. https://doi.org/10.1007/s00181-009-

\section{$\underline{0255-0}$}

Onumah EE, Brümmer B, Hörstgen Schwark G (2010) Elements Which Delimitate Technical Efficiency of Fish Farms in Ghana. Journal of the World Aquaculture Society 41(4):506-518. https://doi.org/10.1111/j.1749-7345.2010.00391.x 
702

703

Pascoe S, Herrero I (2004) Estimation of a composite fish stock index using data envelopment analysis. Fisheries Research 69(1):91-105. https://doi.org/10.1016/j.fishres.2004.03.003

Pastor JT, Lovell CK (2005) A global Malmquist productivity index. Economics Letters 88(2):266-271. https://doi.org/10.1016/j.econlet.2005.02.013

Pipitone V, Colloca F (2018) Recent trends in the productivity of the Italian trawl fishery: The importance of the socio-economic context and overexploitation. Marine Policy 87:135-140. https://doi.org/10.1016/j.marpol.2017.10.017

Rasmus N (2011) GREEN AND TECHNICAL EFFICIENT GROWTH IN DANISH FRESH WATER AQUACULTURE. Aquaculture Economics \& Management 15(4): 262-277. https://doi.org/10.1080/13657305.2011.624574

Sharma KR, Leung PS, Chen H, Peterson A (1999) Economic efficiency and optimum stocking densities in fish polyculture: an application of data envelopment analysis (DEA) to Chinese fish farms. Aquaculture 180(3):207-221. https://doi.org/10.1016/S0044-8486(99)00202-1

Sharma KR, Leung P (2000) Technical efficiency of carp pond culture in South Asia: An application of a stochastic meta-production frontier model. Aquaculture Economics $\&$ Management 4(3-4):169-189. https://doi.org/10.1080/13657300009380268

Simar L, Wilson PW (1998) Sensitivity Analysis of Efficiency Scores: How to Bootstrap in Nonparametric Frontier Models. Management Science 44(1):49-61. https://doi.org/10.1287/mnsc.44.1.49 
Song ML, Zheng WP (2016) Computational analysis of thermoelectric enterprises' environmental efficiency and Bayesian estimation of influence factors. The Social Science Journal

(Fort

Collins) 53(1):88-99. https://doi.org/10.1016/j.soscij.2015.10.002

Terje V, Helen MSH (2011) Technical Progress and Regress in Norwegian Salmon Farming: A Malmquist Index Approach. Marine Resource Economics 26(4):329_ 341. https://doi.org/10.5950/0738-1360-26.4.329

Tingley D, Pascoe S, Coglan L (2005) Factors affecting technical efficiency in fisheries: stochastic production frontier versus data envelopment analysis approaches. Fisheries Research 73(3):363-376. https://doi.org/10.1016/j.fishres.2005.01.008

Troell M, Naylor RL, Metian M, Beveridge M, Tyedmers PH, Folke C, Arrow KJ, Barrett S, Crepin AS, Ehrlich P, Gren R, Kautsky N, Levin SA, Nyborg K, Osterblom H, Polasky S, Scheffer M, Walker BH, Xepapadeas T, Zeeuw de A (2014) Does aquaculture add resilience to the global food system? Proceedings of the National Academy of Sciences - PNAS 111(37):13257-13263. https://doi.org/10.1073/pnas.1404067111

Xu JJ (2020) Research on the efficiency of Marine aquaculture production in China's coastal provinces based on the DEA-Malmquist index. Journal of Guizhou Provincial Party School 01:57-68. http://doi.org/10.16436/j.cnki.525023/d.2020.01.008(in Chinese)

Yang J, Cheng JX, Huang SJ (2020) CO2 emissions performance and reduction potential in China's manufacturing industry: A multi-hierarchy meta-frontier 
approach. Journal of Cleaner Production 255:120226.

748 Yu X, Hu QG, Shen MH (2020) Provincial Differences and Dynamic Changes in Mariculture Efficiency in China: Based on Super-SBM Model and Global

750 Malmquist Index. Biology (Basel, Switzerland) 9(1):18.

751 https://doi.org/10.3390/biology9010018

752 Zhong S, Wang HL, Wen HM, Li J (2020) The total factor productivity index of science and technology innovations in the coastal regions of China between 2006 and 2016. Environmental Science and Pollution Research International. https://doi.org/10.1007/s11356-020-09300-3 
Table 1

Abbreviations used in this paper

\begin{tabular}{ll} 
Abbreviation & Full-name \\
\hline DDF & Directional distance function \\
DEA & Data envelopment analysis \\
DMU & Decision-making unit \\
EC & Technical efficiency change \\
MMI & Meta-frontier Malmquist index \\
MI & Malmquist index \\
R\&D & Research and development \\
SBM & Slack-based measurement \\
TC & Technology change \\
TFP & Total factor productivity \\
TGR & Technology gap ratio \\
\hline
\end{tabular}

Table 2

Variables of freshwater aquaculture efficiency evaluation

\begin{tabular}{lll}
\hline Variables type & Variables & Unit \\
\hline & Freshwater aquaculture professionals & person \\
Input variables & Freshwater seedling quantity & million tail \\
& Freshwater aquaculture area & hectare \\
& The fishery production value of freshwater & 10,000 yuan \\
\hline
\end{tabular}


Figures

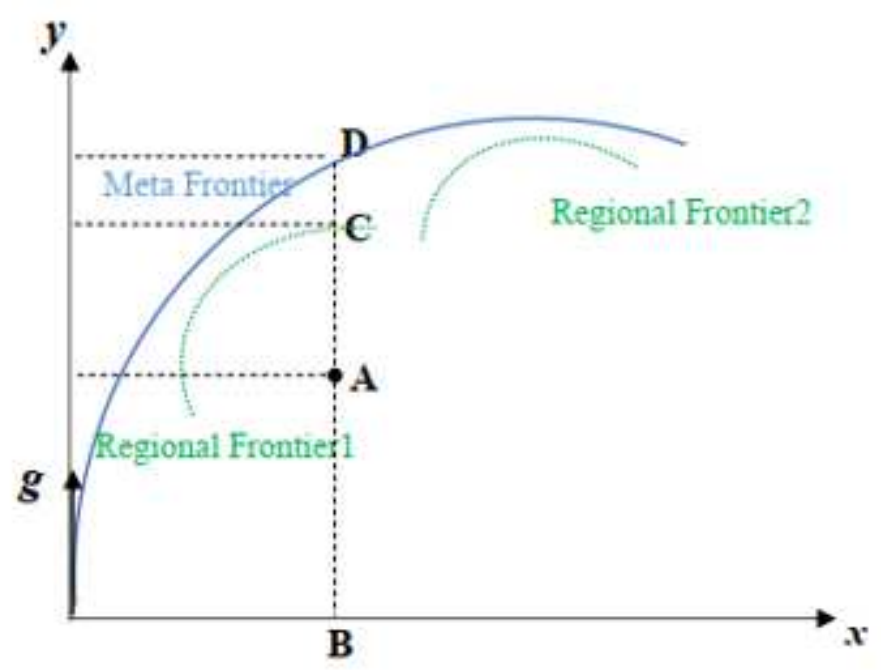

Figure 1

Double-hierarchy meta-frontier
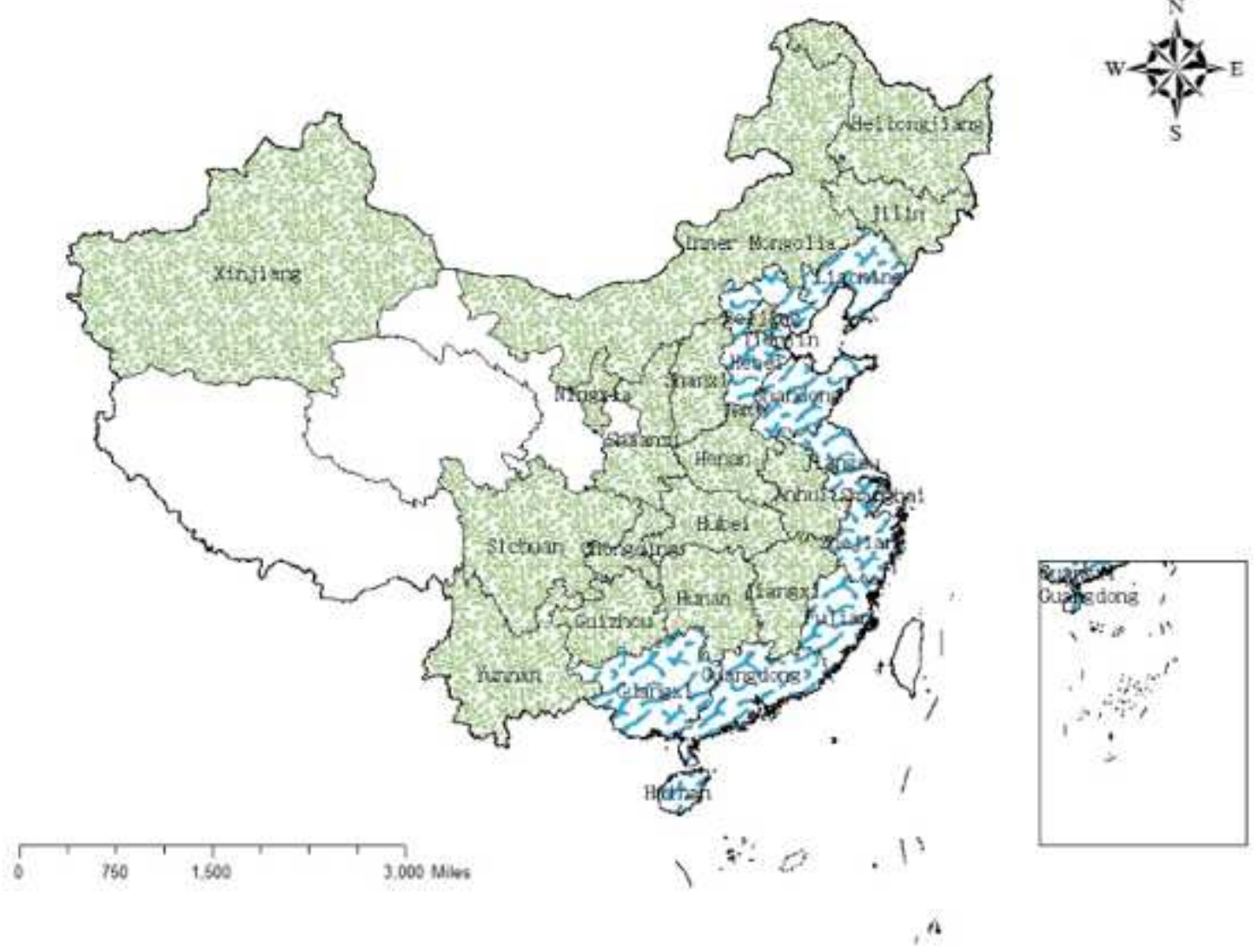

Figure 2 
Spatial distribution sampling in freshwater aquaculture areas. Note: The designations employed and the presentation of the material on this map do not imply the expression of any opinion whatsoever on the part of Research Square concerning the legal status of any country, territory, city or area or of its authorities, or concerning the delimitation of its frontiers or boundaries. This map has been provided by the authors.
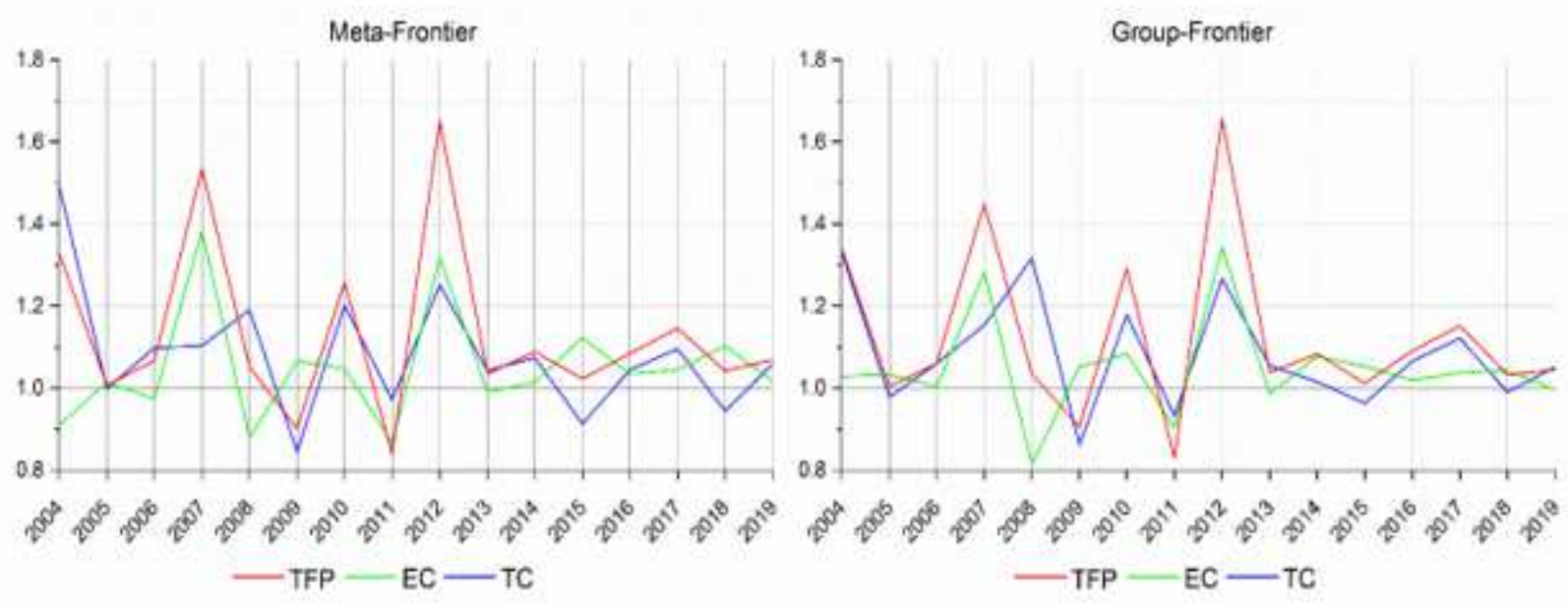

\section{Figure 3}

The trends of TFPIEC and TC at two frontiers 
(1) Average of THP of freshwater aquaculture in meta frontier of

China' s 28 provinces in 2004-2019

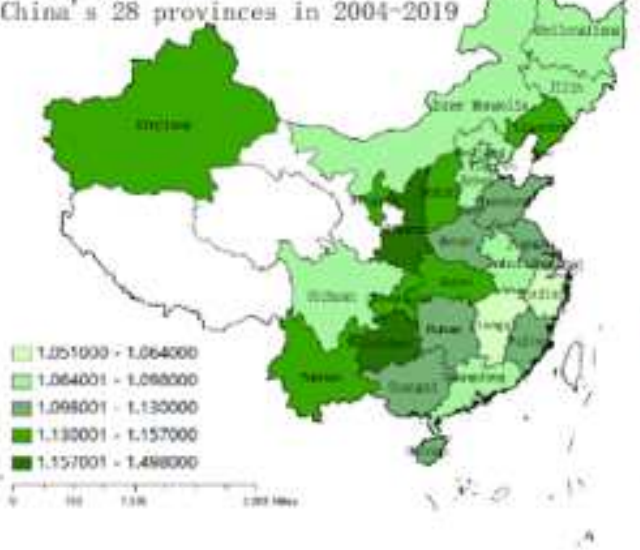

(III) Average of TFP of freshwater equatculture in group-front ier of China's 28 provinces in $2004-2019$

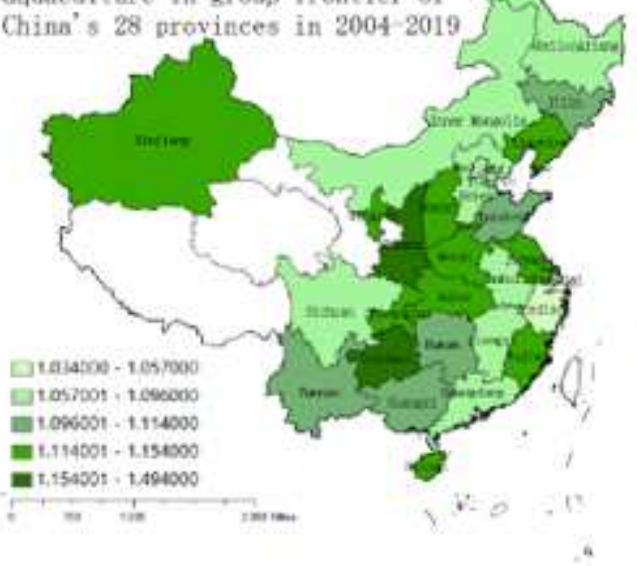

(II) Top and last five of Freshwater Aquaculture in Matefronties

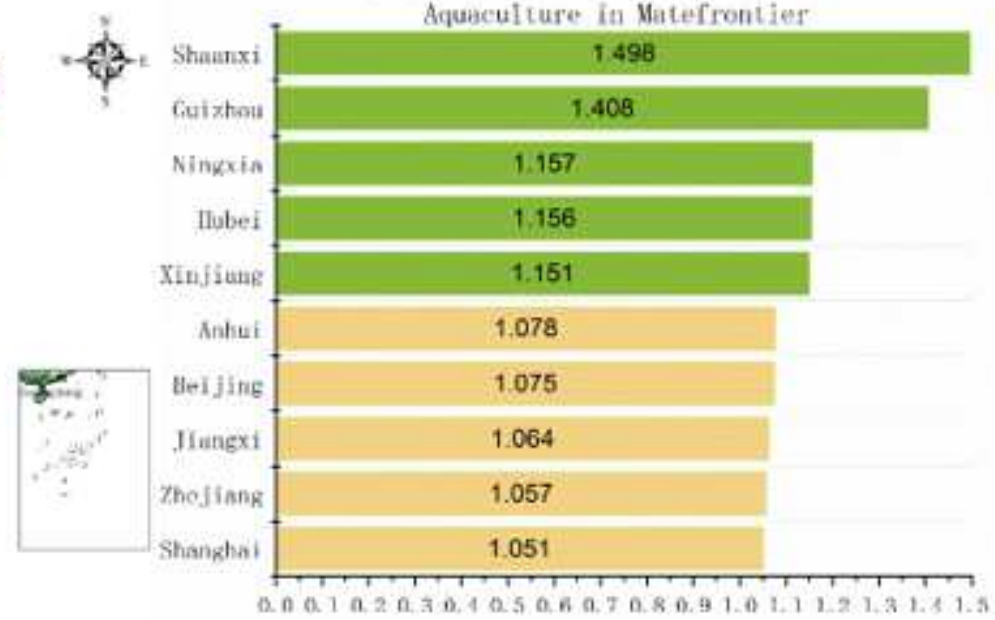

(IV) Top and last rive of Freshwater

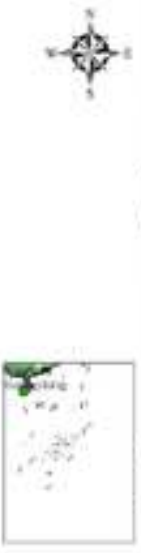

Aquaculture in Groupfrontier

\begin{tabular}{|c|c|}
\hline Shauaxi & 1.494 \\
\hline fuizhot & 1.375 \\
\hline Xinjinge & 1.154 \\
\hline Lieonine & 1.138 \\
\hline Shainxi & 1.131 \\
\hline Sichtuan & 1.076 \\
\hline Jlangx & 1.076 \\
\hline Zhejians & 1.057 \\
\hline Shimentus & 1.05 \\
\hline Heijins & 1.034 \\
\hline
\end{tabular}

\section{Figure 4}

Averages of TFP of freshwater aquaculture in two frontiers. Note: The designations employed and the presentation of the material on this map do not imply the expression of any opinion whatsoever on the part of Research Square concerning the legal status of any country, territory, city or area or of its authorities, or concerning the delimitation of its frontiers or boundaries. This map has been provided by the authors. 


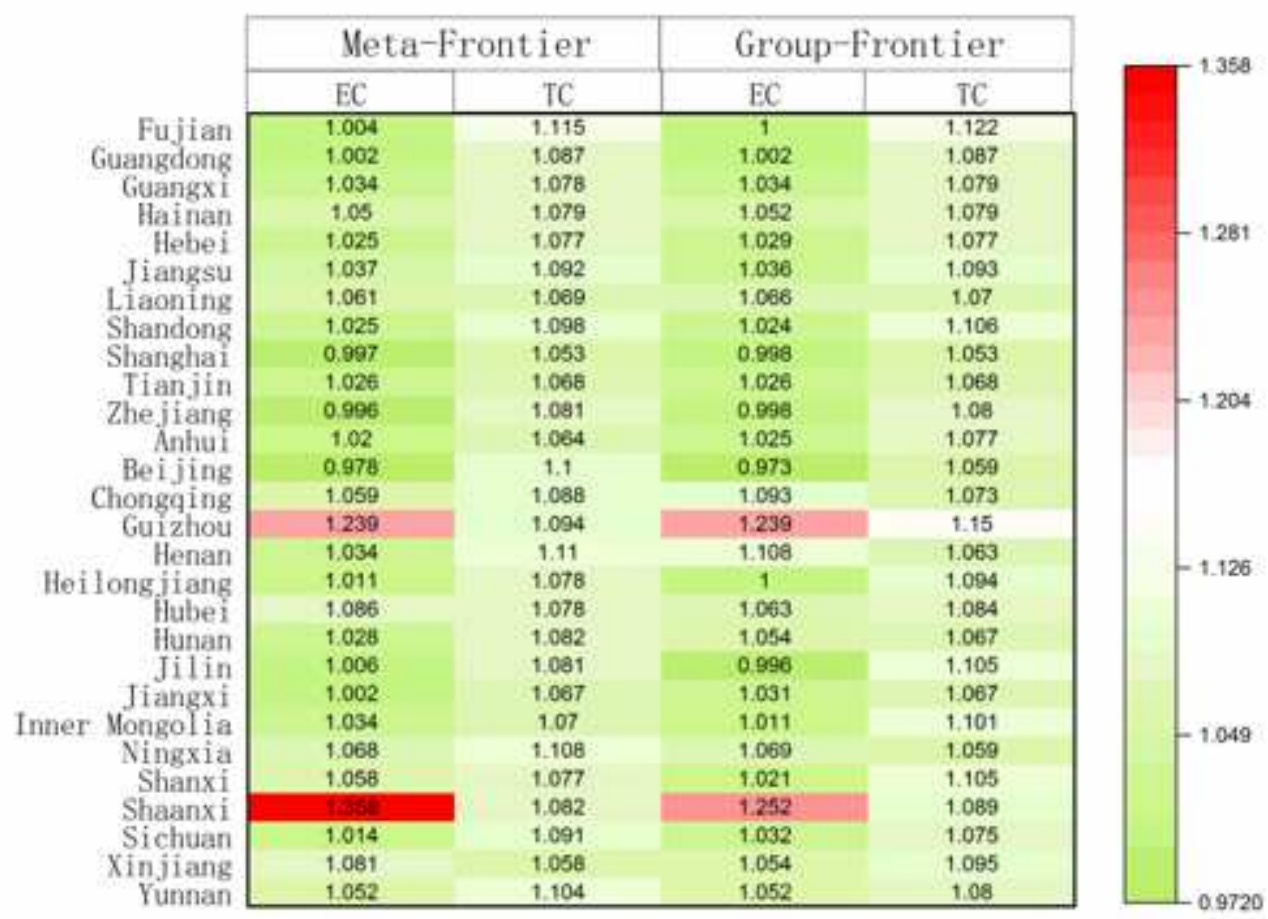

Figure 5

Averages of $\mathrm{EC}$ and $\mathrm{TC}$ of freshwater aquaculture in two frontiers
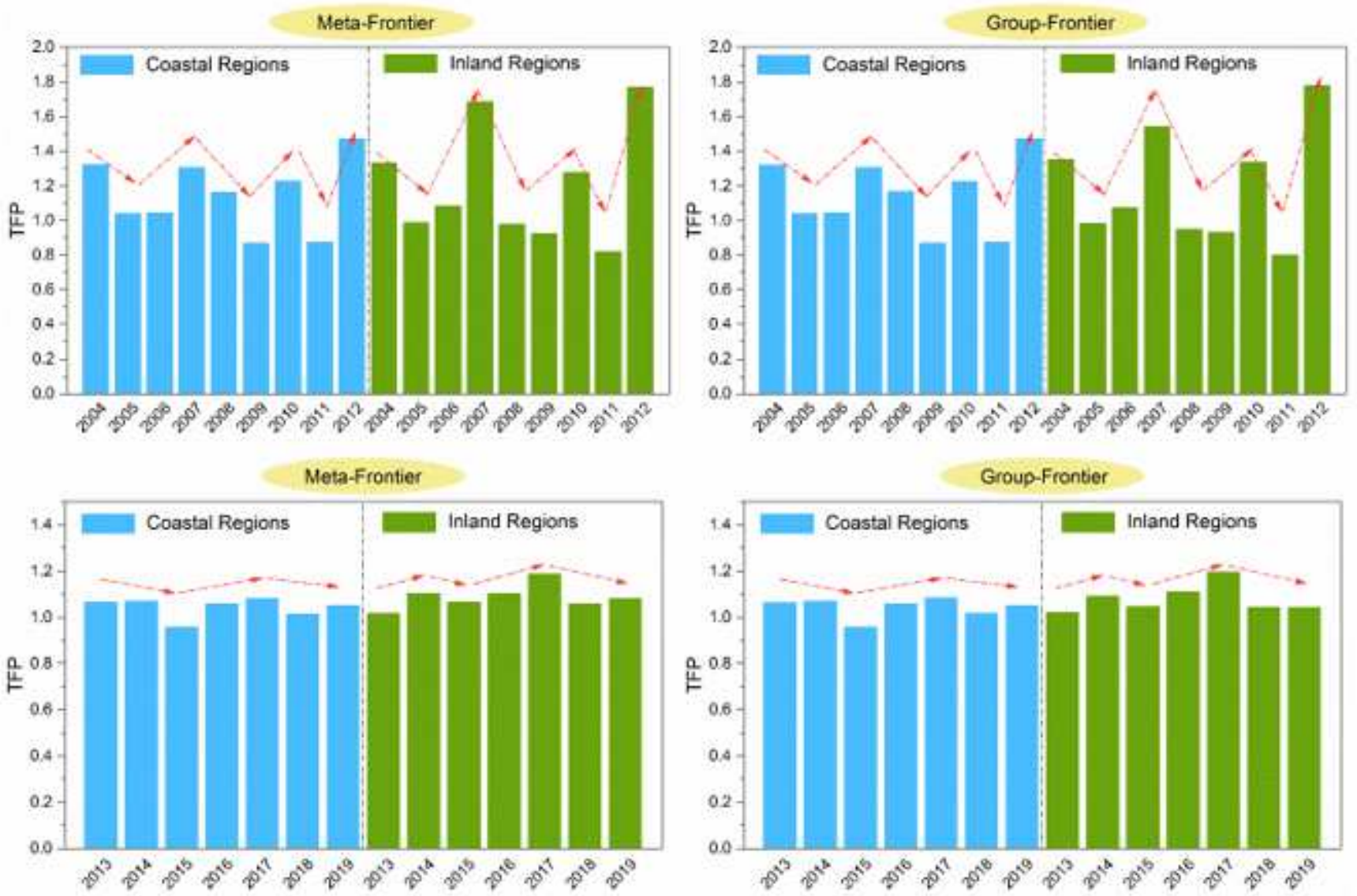
Figure 6

Average annual TFP of freshwater aquaculture in two regions
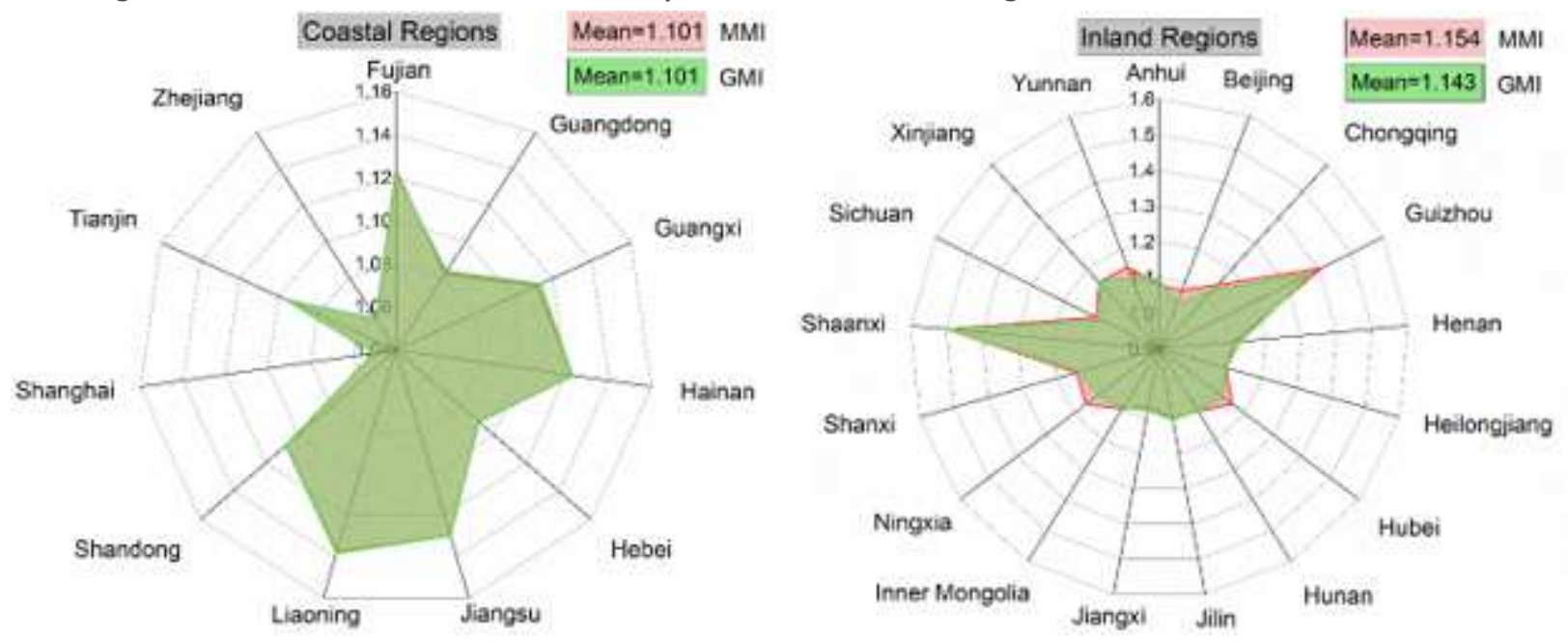

Figure 7

Provinces average TFP of freshwater aquaculture in two regions
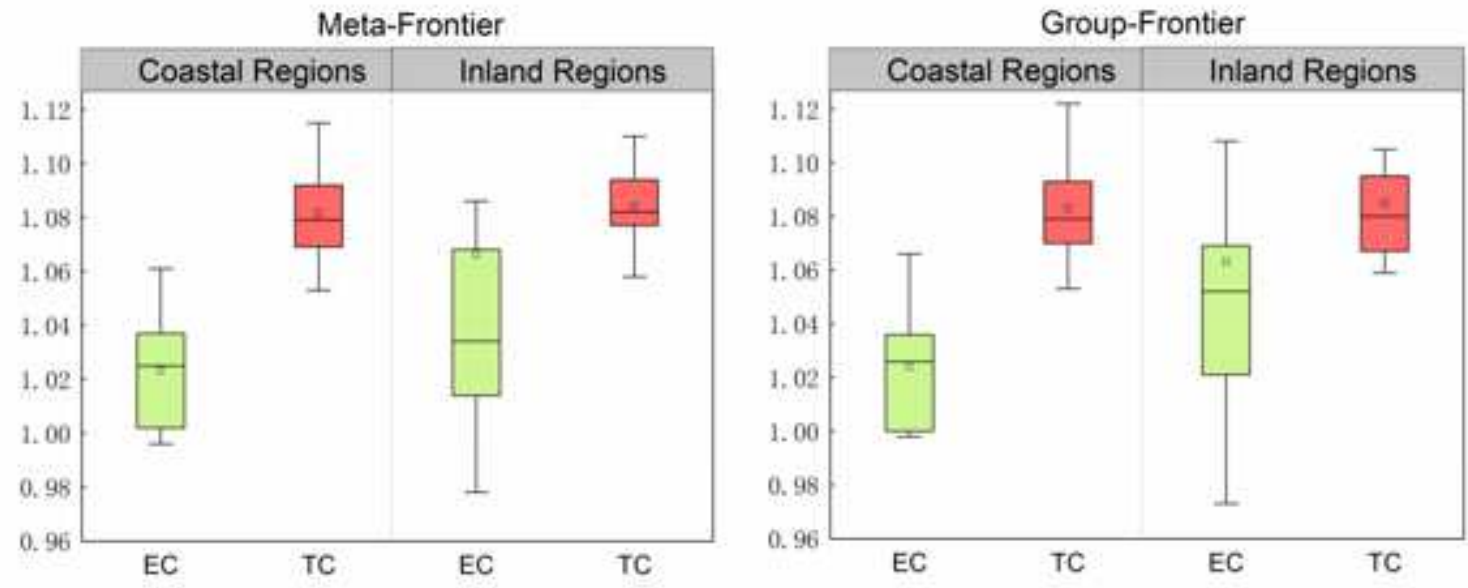

Figure 8

$\mathrm{EC}$ and TC of freshwater aquaculture in two regions 


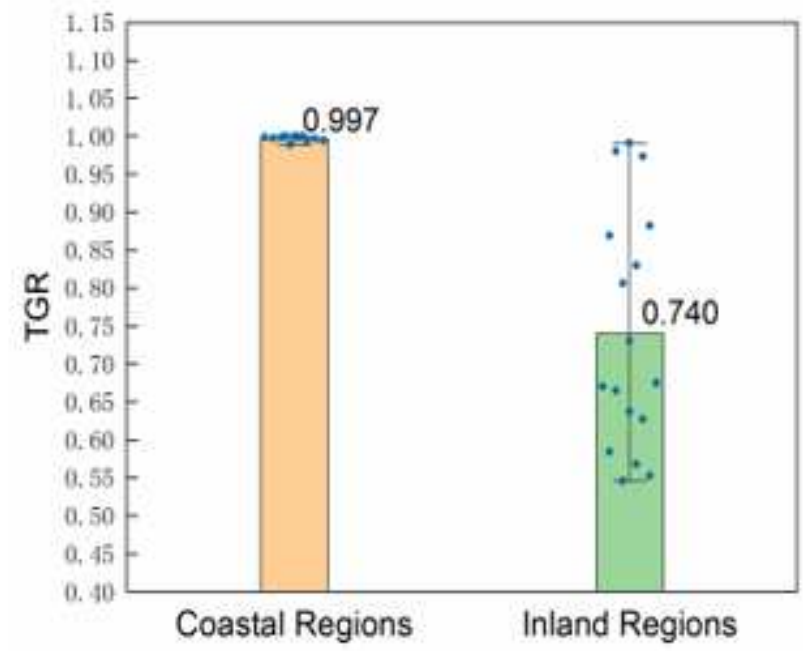

515

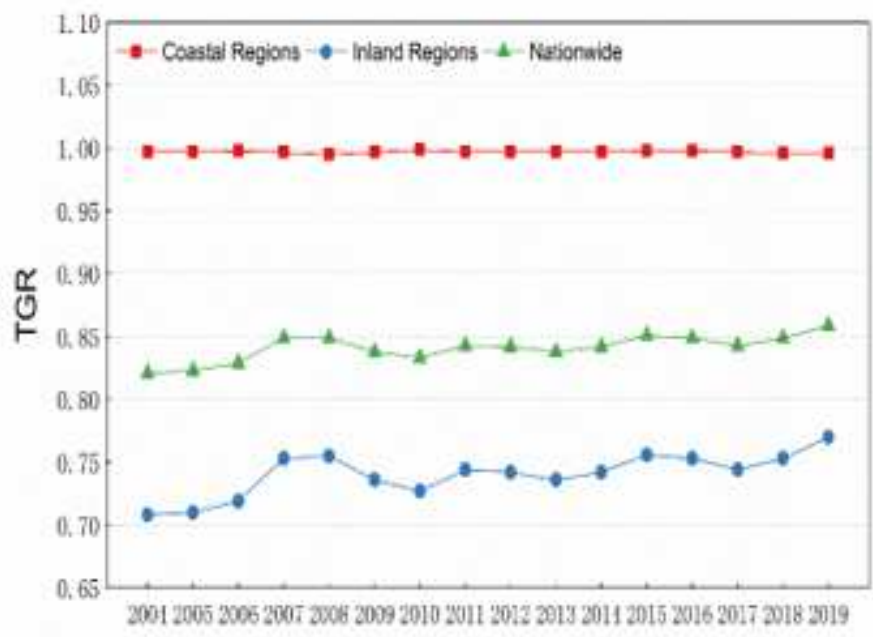

Figure 9

TGR of freshwater aquaculture 